\title{
Support theorems for Funk-type isodistant Radon transforms on constant curvature spaces
}

\author{
Árpád Kurusa ${ }^{1}[$
}

Received: 30 April 2020 / Accepted: 3 September 2021 / Published online: 12 October 2021

(c) The Author(s) 2021

\begin{abstract}
A connected maximal submanifold in a constant curvature space is called isodistant if its points are in equal distances from a totally geodesic of codimension 1 . The isodistant Radon transform of a suitable real function $f$ on a constant curvature space is the function on the set of the isodistants that gives the integrals of $f$ over the isodistants using the canonical measure. Inverting the isodistant Radon transform is severely overdetermined because the totally geodesic Radon transform, which is a restriction of the isodistant Radon transform, is invertible on some large classes of functions. This raises the admissibility problem that is about finding reasonably small subsets of the set of the isodistants such that the associated restrictions of the isodistant Radon transform are injective on a reasonably large set of functions. One of the main results of this paper is that the Funk-type sets of isodistants are admissible, because the associated restrictions of the isodistant Radon transform, we call them Funk-type isodistant Radon transforms, satisfy appropriate support theorems on a large set of functions. This unifies and sharpens several earlier results for the sphere, and brings to light new results for every constant curvature space.
\end{abstract}

Keywords Radon transform · Funk transform · Isodistant - Admissibility · Slice transform · Funk-type transforms $\cdot$ Constant curvature spaces

Mathematics Subject Classification Primary: 44A12 - Secondary: 53C65 · 51M10 · 37E10

\section{Introduction}

Given a totally geodesic $\mathcal{G}$ of codimension 1 in a constant curvature space $\mathbb{K}_{\kappa}^{n}$ of dimension $n \in \mathbb{N}_{2 \leq}$ and of curvature $\kappa \in\{1,0,-1\}$, a connected maximal submanifold $\mathcal{D}$ whose points have a fix distance $\varrho \geq 0$ from $\mathcal{G}$, the axis, is called an isodistant of radius $\varrho$. In the

Research was supported by NFSR of Hungary (NKFIH) under grants K 116451 and KH_18 129630, and by the Ministry for Innovation and Technology of Hungary (MITH) under grant NKFIH-1279-2/2020 and TUDFO/47138-1/2019-ITM.

Árpád Kurusa

kurusa@math.u-szeged.hu

1 Bolyai Institute, University of Szeged, Aradi vértanúk tere 1, 6725 Szeged, Hungary 
constant curvature planes the isodistants are well known, they are the straight lines in the plane, the circles in the sphere, and the hypercycles in the hyperbolic plane [40].

We denote the set of the isodistants by $\mathbb{E}_{\kappa}$, and its subset, the set of the totally geodesics of codimension 1 , by $\mathbb{G}_{K}$.

The isodistant Radon transform $\mathrm{R}_{K}^{\mathbb{E}}$ of a suitable function $f$ on $\mathbb{K}_{K}^{n}$ is defined as the function $\mathrm{R}_{K}^{\mathbb{E}} f$ on $\mathbb{E}_{K}$ that gives the integral of $f$ over every isodistant using the natural measure. The totally geodesic Radon transform $\mathrm{R}_{K}^{\mathbb{G}}$ of a suitable function $f$ on $\mathbb{K}_{K}^{n}$ is defined as the function $\mathrm{R}_{\kappa}^{\mathbb{G}} f$ on $\mathbb{G}_{\kappa}$ that gives the integral of $f$ over every totally geodesic using the natural measure.

The isodistant Radon transform $R_{K}^{\mathbb{E}}$ is injective on a large class of functions, because the totally geodesic Radon transform $R_{\kappa}^{\mathbb{G}}$, which is a restriction of the isodistant Radon transform, is injective by [24, Theorem 3.2]. This shows that the inversion problem of the isodistant Radon transform is severely overdetermined, and hence, the admissibility problem $[15,17]$ arises:

What are the reasonably small submanifolds of $\mathbb{E}_{\kappa}$ for which

the restricted isodistant Radon transform is injective on

a reasonably large space of functions?

We call such a submanifold of $\mathbb{E}_{K}$ admissible ${ }^{1}[15,17]$. For instance $\mathbb{G}_{K}$ is an admissible submanifold of $\mathbb{E}_{K}[24$, Theorem 3.2].

Let the hypersurface $\mathcal{K}_{\kappa}^{n} \subset \mathbb{R}^{n+1}$ of points $\boldsymbol{p}=\left(p_{1}, \ldots, p_{n}, p_{n+1}\right)$ satisfying

$$
\kappa\left(p_{1}^{2}+\cdots+p_{n}^{2}\right)+p_{n+1}^{2}=1
$$

be equipped with the Riemannian metric

$$
g_{\kappa ; \boldsymbol{p}}: T_{\boldsymbol{p}} \mathcal{K}_{\kappa}^{n} \times T_{\boldsymbol{p}} \mathcal{K}_{\kappa}^{n} \ni(\boldsymbol{x}, \boldsymbol{y}) \mapsto x_{1} y_{1}+\cdots+x_{n} y_{n}+\kappa x_{n+1} y_{n+1}
$$

at every point $\boldsymbol{p} \in \mathcal{K}_{\kappa}^{n}$. Then one gets the so-called projective model $\overline{\mathcal{K}}_{\kappa}^{n}$ of the constant curvature space $\mathbb{K}_{K}^{n}[10]$, and also the canonical correspondence

$$
\chi_{\kappa}: \mathcal{K}_{\kappa}^{n} \ni E \rightarrow\{E,-E\} \in \overline{\mathcal{K}}_{\kappa}^{n} \cong \mathbb{K}_{\kappa}^{n}
$$

by identifying the points of $\mathcal{K}_{\kappa}^{n} \subset \mathbb{R}^{n+1}$ that are symmetric in the origin.

It is very well known that every 1-codimensional totally geodesic of $\mathbb{K}_{\kappa}^{n}$ is the intersection of $\overline{\mathcal{K}}_{\kappa}^{n}$ with a 1-codimensional subspace of $\mathbb{R}^{n+1}$ [24]. It is less known (see Lemma 3.1) that every isodistant of $\mathbb{K}_{\kappa}^{n}$ corresponds to a slice, i.e., a hyperplane section of $\mathcal{K}_{\kappa}^{n}$.

The slice transform $\mathrm{S}^{\kappa}$ of a suitable real function $f$ on $\mathcal{K}_{\kappa}^{n}$ is defined as the function $S^{\kappa} f$ on the set of slices that gives the integral of $f$ over every slice using the canonical measure. After giving explicit formulas for $S^{\kappa}$ in Sect. 4, we prove intertwining relations between the slice transforms and the classical Euclidean Radon transform in Sect. 5.

We call a set of slices rotational if it contains all of its rotations about the $(n+1)$ th axis. The set of the hyperplanes of the slices in a rotational set of slices is clearly rotation invariant, so they pass through a common point $P=(0, \ldots, 0, p)$ of the $(n+1)$ th axis, hence they are determined by the tangent $q=\tan \alpha \in[0, \infty]$ of the angle $\alpha$ the hyperplanes closes with

${ }^{1}$ We specify the space of the applicable functions only when it is important for some reason. 
the $(n+1)$ th axis. The pairs $(p, q)$ form a subset of the upper half plane extended with ideal points. So, the admissibility problem for the rotational slice transform can be formulated as to

\section{determine the curves $\mathcal{C}$ in the $(p, q)$ plane (equipped with ideal elements) such that the slice transform associated with the rotational set of slices given by $\mathcal{C}$ is injective on a reasonably large set of functions.}

We call these curves admissible. Some curves are known to be admissible or inadmissible. For $\kappa=-1$, the straight line $q=1$ belongs to the horocyclic Radon transform [8, 9, 19, 22, 29], and so it is admissible. For $\kappa=1$, the hyperbola $r^{2}\left(1+q^{2}\right)=p^{2} q^{2}(r \in(0,1))$ belongs to the Radon transform associated with the subspheres of radius $\sqrt{1-r^{2}}$, and so, by [36], it is admissible if and only if $r$ is not a root of any Gegenbauer polynomial of the weight $\left(1-x^{2}\right)^{\frac{n-3}{2}}$. For $\kappa=1$, the curve $1=p^{2}-q^{2} \cosh ^{2} \lambda(\lambda \in[0, \infty])$ belongs to the Radon transform associated with the subspheres whose hyperplanes are tangent to the spheroid $1=\left(x_{1}^{2}+\cdots+x_{n}^{2}\right) \cosh ^{2} \lambda+x_{n+1}^{2}$, so, by [35], it is admissible.

If $\mathcal{C}$ is a ray with fixed $p \in \mathbb{R} \cup\{ \pm \infty\}$, then we call the associated restrictions of the slice transform $p$-shifted Funk transform ${ }^{2}$ and denote it by $\mathrm{F}_{p}^{\kappa}$. For the sphere $(\kappa=1)$ this was recently quite intensively investigated $[3,4,6,7,18,20,25,26,30,32-34,38]$, but there are also sporadic earlier results $[1,16]$ as well. Surprisingly enough there seems to be no general results for $\kappa=0,-1$.

The most important examples of the $p$-shifted Funk transforms are the Funk transform $\mathrm{F}_{0}^{1}[13]$ and the spherical slice transforms ${ }^{3} \mathrm{~F}_{ \pm 1}^{1}[1,18,32,34]$, and their hyperbolic counterparts $\mathrm{F}_{0}^{-1}$ and $\mathrm{F}_{ \pm 1}^{-1}$, the hyperbolic Funk transform and hyperbolic slice transforms that are introduced here.

We prove sharp support theorems and explicit kernel descriptions for every $\mathrm{F}_{p}^{\kappa}$ for each $\kappa \in\{0, \pm 1\}$ in Sect. 6, where the main tool is the intertwining relations, (5.4) and (5.5), of the slice transform and the Euclidean Radon transform. It is interesting, that, depending on $p$, different speeds of decay on the functions are necessary to employ for the support theorems.

We define the Funk-type isodistant Radon transform $\hat{\mathrm{R}}_{p}^{\kappa}$ of a suitable function $h$ on $\mathbb{K}_{\kappa}^{n}$ as the shifted Funk transform of $\hat{h}:=J \cdot h \circ \chi_{\kappa}$, where $J$ is the indicator function of the open upper half space of $\mathbb{R}^{n+1}$. It is considered in Sect. 7, where again sharp support theorems and complete kernel descriptions are proved. These results considerably generalize the author's earlier support theorems [24] for the totally geodesic Radon transform.

In $\mathbb{K}_{\kappa}^{n}$ every 1-codimensional totally geodesic has exactly two isodistants for every $\varrho>0$. We call the union of such a pair of isodistants a duplex isodistant and define the duplex Funk-type isodistant Radon transform $\mathrm{R}_{p}^{\kappa}$ of a suitable function $h$ on $\mathbb{K}_{\kappa}^{n}$ as the shifted Funk transform of $\tilde{h}:=h \circ \chi_{\kappa}$. It is considered in Sect. 8, where again sharp support theorems and complete kernel descriptions are proved. When $\kappa=1$, these results give geometric reasoning for [5,6]. For $\kappa=0$ we do not get too much new, but we observe a new kind of problem that is discussed and solved in a special case in Sect. 9.

\footnotetext{
2 This term follows the phrasing used by [5].

3 We use this term of [18] and even use the analogous phrase for the hyperbolic case although it breaks our terminology a little bit.
} 
The presented support theorems and kernel descriptions are new for both curved constant curvature spaces. Further, these results bring to light new problems for all constant curvature spaces that we discuss in last Sect. 9 where some possible generalizations, consequences and worthy details are also outlined.

\section{Notations and preliminaries}

Points of $\mathbb{R}^{n}$ are denoted as $A, B, \ldots$ or $\boldsymbol{a}, \boldsymbol{b}, \ldots$, and vectors are given as $\overrightarrow{A B}$ or $\boldsymbol{a}, \boldsymbol{b}, \ldots$ The straight line through $A$ and $B$ is $A B$, and the closed segment with endpoints $A$ and $B$ is $\overline{A B}$.

We denote the Euclidean scalar product by $\langle\cdot, \cdot\rangle, \mathcal{B}^{n}$ is the $n$-dimensional closed unit ball centered at the origin, and its boundary is $\mathcal{S}^{n-1}=\partial \mathcal{B}^{n}$. If $n=2$, then we use the notation $\boldsymbol{u}_{\alpha}=(\cos \alpha, \sin \alpha)$ for the elements of $\mathcal{S}^{1}$.

We parameterize the manifold of the hyperplanes, the 1-codimensional totally geodesics in $\mathbb{R}^{n+1}$, on $\mathcal{S}^{n} \times \mathbb{R}$, so that $\mathcal{P}(\boldsymbol{w}, r)=\{\boldsymbol{x}: r=\langle\boldsymbol{w}, \boldsymbol{x}\rangle\}$. This is a double covering, but it will not cause trouble. Then we have

$$
\mathcal{P}(\boldsymbol{w}, r)=\left\{\frac{r}{\langle\boldsymbol{w}, \boldsymbol{u}\rangle} \boldsymbol{u}: \boldsymbol{u} \in \mathcal{S}^{n},\langle\boldsymbol{w}, \boldsymbol{u}\rangle>0\right\} \quad \text { if } r \neq 0,
$$

so the classical Euclidean Radon transform $\mathrm{R}[18,28]$ on the set of suitable functions on $\mathbb{R}^{n}$ is defined [24, (2.4)], for $r>0$, by

$$
\mathrm{R} f(\boldsymbol{w}, r)=\mathrm{R}_{0}^{\mathbb{G}} f(\mathcal{P}(\boldsymbol{w}, r))=\frac{1}{r} \int_{\mathcal{S}_{w, 0}^{n-1}} f\left(\frac{r}{\langle\boldsymbol{w}, \boldsymbol{u}\rangle} \boldsymbol{u}\right)\left(\frac{r}{\langle\boldsymbol{w}, \boldsymbol{u}\rangle}\right)^{n} \mathrm{~d} \boldsymbol{u},
$$

where $\boldsymbol{w} \in \mathcal{S}^{n-1}$, and $\mathcal{S}_{w, s}^{n-1}=\left\{\boldsymbol{u} \in \mathcal{S}^{n-1}:\langle\boldsymbol{w}, \boldsymbol{u}\rangle>s\right\}(s \in \mathbb{R})$. Let $C_{\infty}\left(\mathbb{R}^{n}\right)$ be the space of all continuous functions $f$ on $\mathbb{R}^{n}$ such that $f(\boldsymbol{x})|\boldsymbol{x}|^{k}$ is bounded for each $k>0$ (this is a special case of (6.6)). Then we have the following support theorem of Helgason that is crucial for our results.

Theorem 2.1 ([18, Theorem 2.6 of Chapter I]) If $f \in C_{\infty}\left(\mathbb{R}^{n}\right)$, and there exists a constant $A>0$ such that $\operatorname{Rf}(\mathcal{P})$ vanishes for every hyperplane farther from the origin than $A$, then $f(\boldsymbol{x})=0$ for $|x|>A$.

Notice that counter examples show that the decay condition in this theorem cannot be dropped (see [18, Remark 2.9 of Chapter I] and also [28, pp. 233-235].).

We fix the vectors $\boldsymbol{b}_{i}=\left(\delta_{i, 1}, \ldots, \delta_{i, n}, \delta_{i, n+1}\right)$, where $\delta_{i, j}$ is the Kronecker-delta and $i, j=1, \ldots, n+1$, and denote the hyperplane of equation $x_{n+1}=p \in \mathbb{R}$ by $\mathcal{A}_{p}^{n}$. We define the projections $\Pi_{p}$ from $\mathbb{R}^{n+1} \backslash \mathcal{A}_{p}^{n}$ by

$$
\Pi_{p}\left(x_{1}, \ldots, x_{n}, x_{n+1}\right)= \begin{cases}\left(\frac{x_{1}}{x_{n+1}-p}, \ldots, \frac{x_{n}}{x_{n+1}-p}, p+1\right) & \text { if } p \in \mathbb{R}, \\ \left(x_{1}, \ldots, x_{n}, \pm \infty\right) & \text { if } p= \pm \infty\end{cases}
$$

and introduce $O=(0, \ldots, 0), O^{+}=(0, \ldots, 0,1), O^{-}=(0, \ldots, 0,-1)$, 


$$
\hat{\mathcal{K}}_{\kappa}^{n}=\mathcal{K}_{\kappa}^{n} \cap\left\{\boldsymbol{p}:\left\langle\boldsymbol{p}, \boldsymbol{b}_{n+1}\right\rangle>0\right\}, \quad \check{\mathcal{K}}_{\kappa}^{n}=\mathcal{K}_{\kappa}^{n} \backslash \hat{\mathcal{K}}_{\kappa}^{n}, \text { and } \tilde{\mathcal{K}}_{\kappa}^{n}= \begin{cases}\mathcal{K}_{1}^{n} & \text { if } \kappa=1, \\ \hat{\mathcal{K}}_{\kappa}^{n} & \text { otherwise. }\end{cases}
$$

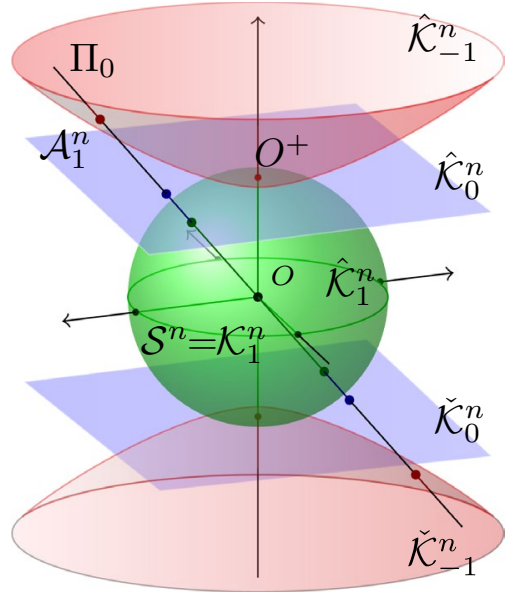

Restricting $\Pi_{0}$ to $\mathcal{K}_{\kappa}^{n}$ essentially gives the so-called gnomonic projection that results in the so-called projective models, i.e., the Cayley-Klein models of the constant curvature spaces.

The domain $\overline{\mathcal{M}}_{\kappa ; 1}^{n}$ of such a Cayley-Klein model, is $\mathcal{A}_{1}^{n}$ with the ideal hyperplane if $\kappa=1,0$, and it is the interior of the unit ball centered to $O^{+}$in $\mathcal{A}_{1}^{n}$ if $\kappa=-1$. The geodesics are the chords of $\overline{\mathcal{M}}_{\kappa ; 1}^{n}$, the totally geodesics are the $n$-dimensional slices (hyperplanes) of $\mathcal{B}^{n} \subset \mathcal{A}_{1}^{n}[10]$, hence every totally geodesic of $\overline{\mathcal{K}}_{\kappa}^{n}$ is the intersection of $\overline{\mathcal{K}}_{\kappa}^{n}$ with a 1-codimensional subspace of $\mathbb{R}^{n+1}$ [24].

The manifold $\mathcal{K}_{\kappa}^{n}$ is a rotational one [21], so it is determined by the size function $\sigma_{\kappa}$ giving the radius $\sigma_{K}(r)$ of the Euclidean sphere that is isometric with the geodesic sphere of radius $r$ in $\mathcal{K}_{\kappa}^{n}$. This defines the function $\eta_{\kappa}(\cdot)=\sqrt{1-\kappa \sigma_{\kappa}^{2}(\cdot)}$, while the projector function $\tau_{\kappa}[24]$ is defined by $\Pi_{0}(r w)=\tau_{\kappa}(r) w$.

We often use the polar coordinatization of $\hat{\mathcal{K}}_{\kappa}^{n}$ and $\check{\mathcal{K}}_{\kappa}^{n}$ with respect to the appropriate point $O^{ \pm}$: the pair $(\boldsymbol{u}, r)$ means the point $\operatorname{Exp}_{O^{ \pm}}(r \boldsymbol{u})$, where $\boldsymbol{u} \in \mathcal{S}^{n-1} \subset T_{O^{ \pm}} \mathcal{K}_{\kappa}^{n}$ is a unit vector, $r \in \mathbb{R}_{+}$, and $\operatorname{Exp}$ is the usual exponential mapping, hence $d_{\kappa}\left(O^{ \pm}, \operatorname{Exp}_{O^{ \pm}}(r \boldsymbol{u})\right)=r$ for the metric $d_{\kappa}$ on $\mathcal{K}_{\kappa}^{n}$ determined by (1.2). The injectivity radius $\imath_{\kappa}>0$ is then the upper limit of the second parameter until which the polar coordinatization keeps injectivity. Finally, the supremum $\rho_{\kappa}>0$ of the distances a point can be from a geodesic is called the geodesic injectivity radius (Table 1).

Table 1 Properties of constant curvature spaces

\begin{tabular}{lllllll}
\hline Spaces (type) & $\kappa$ & $\sigma_{\kappa}$ & $\tau_{\kappa}$ & $\eta_{\kappa}$ & $\rho_{\kappa}$ & $l_{\kappa}$ \\
\hline$\hat{\mathcal{K}}_{-1}^{n}$ (hyperbolic) & -1 & $\sinh$ & $\tanh$ & $\cosh$ & $\infty$ & $\infty$ \\
$\hat{\mathcal{K}}_{0}^{n}$ (Euclidean) & 0 & Id & Id & 1 & $\infty$ & $\infty$ \\
$\mathcal{K}_{1}^{n}$ (spherical) & +1 & $\sin$ & $\tan$ & $\cos$ & $\pi / 2$ & $\pi$ \\
\hline
\end{tabular}




\section{Isodistants and hyperplanes}

We parameterize the manifold $\tilde{\mathbb{G}}_{\kappa}$ of the totally geodesics of $\tilde{\mathcal{K}}_{\kappa}^{n}$ on $\mathcal{S}^{n-1} \times\left[0, \rho_{\kappa}\right.$ ) so that the totally geodesic $\tilde{\mathcal{G}}(\boldsymbol{w}, g)$ is perpendicular to the geodesic $t \mapsto \operatorname{Exp}_{O^{+}}(t \boldsymbol{w})$ and contains the point $\operatorname{Exp}_{O^{+}}(g \boldsymbol{w})$ (this leaves out $\tilde{\mathcal{K}}_{1}^{n} \cap \mathcal{A}_{0}^{n}$ ), where $\boldsymbol{w} \in \mathcal{S}^{n-1} \subset T_{O^{+}} \tilde{\mathcal{K}}_{\kappa}^{n}$ and $g \in\left[0, \rho_{\kappa}\right)$. This is a double covering at $g=0$, but it will not cause problem. The manifold $\tilde{\mathbb{E}}_{K}$ of the isodistants in $\tilde{\mathcal{K}}_{\kappa}^{n}$ is parameterized on $\mathcal{S}^{n-1} \times\left\{(g, \rho): g \in\left[0, \rho_{\kappa}\right)\right.$ and $\left.\rho+g \in\left(-\rho_{\kappa}, \rho_{\kappa}\right)\right\}$ so that

$\tilde{\mathcal{D}}(\boldsymbol{w}, g ; \varrho)$ is the $\varrho$ - isodistant of the axis $\hat{\mathcal{G}}(\boldsymbol{w}, g) \in \hat{\mathbb{G}}_{\kappa}$ that passes the point $\operatorname{Exp}_{O^{+}}(\boldsymbol{w}, g+\varrho)$.

The following lemma shows that the isodistants are plane sections of $\tilde{\mathcal{K}}_{\kappa}^{n}$.

Lemma 3.1 For any $\boldsymbol{w}=\left(w_{1}, \ldots, w_{n-1}, 0\right) \in \mathcal{S}^{n} \cap \mathcal{A}_{0}^{n}$ we have

$$
\tilde{\mathcal{D}}(\boldsymbol{w}, g ; \rho)=\tilde{\mathcal{K}}_{\kappa}^{n} \cap \mathcal{P}\left(\frac{\boldsymbol{w}-\tau_{\kappa}(g) \boldsymbol{b}_{n+1}}{\sqrt{1+\tau_{\kappa}^{2}(g)}}, \frac{\sigma_{\kappa}(\varrho)}{\sqrt{\eta_{\kappa}^{2}(g)+\sigma_{\kappa}^{2}(g)}}\right) .
$$

Proof Formula (3.1) clearly holds for $\kappa \in\{0,1\}$ so we assume $\kappa=-1$.

Firstly, we determine the point $D$ of $\tilde{\mathcal{K}}_{-1}^{n} \cap \mathcal{P}\left(\frac{w-\tanh g b_{n+1}}{\sqrt{1+\tanh ^{2} g}}, r\right)$, closest to point $O^{+}$, where $r \in \mathbb{R}$. Due to the rotational invariance of $\tilde{\mathcal{K}}_{\kappa}^{n}, D$ is in the 2-dimensional plane spanned by $\boldsymbol{w}$ and $\boldsymbol{b}_{n+1}$. Let $G$ be the point in $\tilde{\mathcal{K}}_{-1}^{n} \cap \mathcal{P}\left(\frac{w-\tanh g \boldsymbol{b}_{n+1}}{\sqrt{1+\tanh ^{2} g}}, 0\right)$ closest to point $O^{+}$. As $G, D \in \tilde{\mathcal{K}}_{-1}^{n}$ we have $G=\sinh g \boldsymbol{w}+\cosh g \boldsymbol{b}_{n+1}$ and $D=\sinh (g+\rho) \boldsymbol{w}+\cosh (g+\varrho) \boldsymbol{b}_{n+1}$ for some $g, \varrho \geq 0$. Let $\ell$ be the line passing $O^{+}$in direction $w$, and let $D^{\perp}$ be the orthogonal projection of $D$ on $\ell$. Let $O^{\perp}$ be the orthogonal projection of $O$ on $\mathcal{P}\left(\frac{w-\tanh g b_{n+1}}{\sqrt{1+\tanh ^{2} g}}, r\right)$. Let $X=\ell \cap O G, Y=\ell \cap O D, Z=\ell \cap O^{\perp} D$. Figure 1 shows what we have.
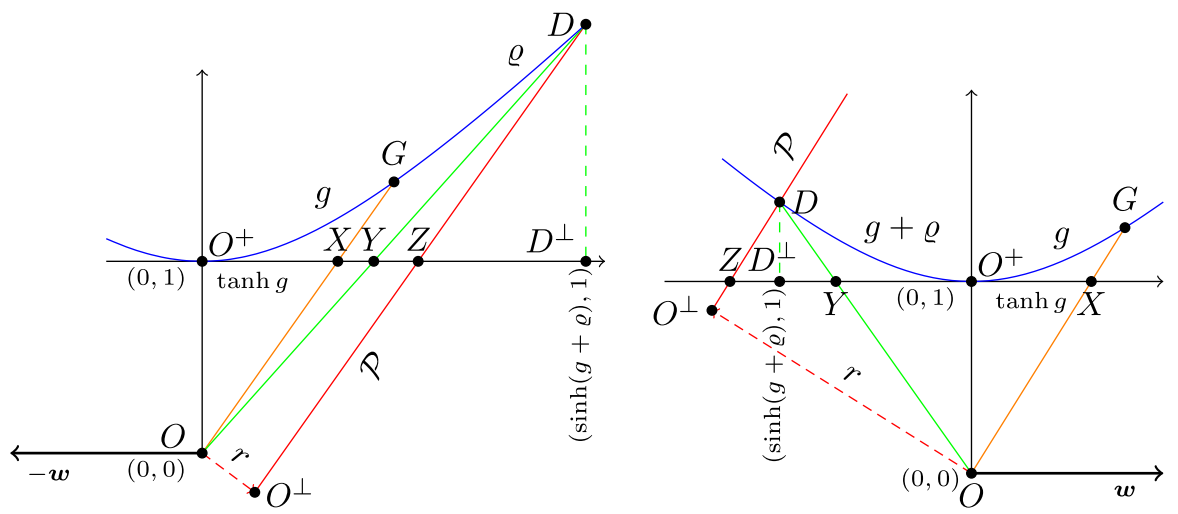

Fig. 1 Depiction of the plane spanned by $\boldsymbol{w}$ and $\boldsymbol{b}_{n+1}$ shows $d\left(D, D^{\perp}\right)=\cosh (g+\varrho)$ for isodistants of positive and negative radius 
We clearly have

$$
d\left(O^{+}, X\right)=\tanh g, \quad d\left(O^{+}, Y\right)=\tanh (g+\varrho), \quad d\left(O^{+}, D^{\perp}\right)=\sinh (g+\varrho) .
$$

Triangles $\triangle(O X Y)$ and $\triangle(D Z Y)$ are similar and the ratio of the similarity is $\frac{D D^{\perp}}{O O^{+}}=\cosh (g+\rho)-1$, so $d(Y, Z)=d(X, Y)(\cosh (g+\rho)-1)$. Thus

$$
d(X, Z)=d(X, Y)+d(Y, Z)=d(X, Y) \cosh (g+\varrho) .
$$

Further, $d(X, Y)=\operatorname{sign}(\varrho)(\tanh (g+\varrho)-\tanh g)$, hence

$$
r=\frac{\tanh (g+\rho)-\tanh g}{\sqrt{1-\tanh ^{2}(g+\rho)} \sqrt{1+\tanh ^{2} g}}=\sinh \rho \frac{\sqrt{1-\tanh ^{2} g}}{\sqrt{1+\tanh ^{2} g}} .
$$

Now we determine the slice $\mathcal{C}:=\tilde{\mathcal{K}}_{-1}^{n} \cap \mathcal{P}\left(\frac{w-\tanh g b_{n+1}}{\sqrt{1+\tanh ^{2} g}}, r\right)$.

For any unit vector $\boldsymbol{w}$ every point of $\mathbb{R}^{n+1}$ can be uniquely written in the form $x \boldsymbol{w}+y \boldsymbol{w}^{\perp}+z \boldsymbol{b}_{n+1}$, where $\boldsymbol{w}^{\perp}$ is a unit vector in the orthogonal complement of the plane spanned by $\boldsymbol{w}$ and $\boldsymbol{b}_{n+1}$.

In this form a point is in $\mathcal{C}:=\tilde{\mathcal{K}}_{-1}^{n}$ if and only if

$$
x^{2}+y^{2}+1=z^{2} \text { and } z \tanh g=x-r \sqrt{1+\tanh ^{2} g}
$$

Since the stereographic projection $\Pi_{-1}$ into the subspace $\mathcal{A}_{0}^{n}$ is

$$
\Pi_{-1}: x w+y w^{\perp}+z b_{n+1} \mapsto \frac{x}{1+z} w+\frac{y}{1+z} w^{\perp}=: s w+t w^{\perp},
$$

we get

$$
\begin{cases}1+z=1+s(1+z) \operatorname{coth} g-r \sqrt{1+\operatorname{coth}^{2} g} & \text { if } g \neq 0, \\ z^{2}=1+r^{2}+y^{2}=1+r^{2}+t^{2}(1+z)^{2} & \text { if } g=0 .\end{cases}
$$

for the points of $\mathcal{C}$. So we can express $1+z$ as

$$
1+z= \begin{cases}\frac{r \sqrt{1+\operatorname{coth}^{2} g}-1}{s \operatorname{coth} g-1}=\frac{r \sqrt{1+\tanh ^{2} g}-\tanh g}{s-\tanh g} & \text { if } g \neq 0 \\ \frac{1+\sqrt{1+r^{2}\left(1-t^{2}\right)}}{1-t^{2}} & \text { if } g=0\end{cases}
$$

hence $\Pi_{-1}(\mathcal{C})$ is the solution of the equation

$$
s^{2}+t^{2}=\frac{z-1}{z+1}= \begin{cases}\frac{r \sqrt{1+\tanh ^{2} g}+\tanh g}{r \sqrt{1+\tanh ^{2} g}-\tanh g}-\frac{2 s}{r \sqrt{1+\tanh ^{2} g}-\tanh g} & \text { if } g \neq 0, \\ \frac{2 t^{2}-\left(1-\sqrt{1+r^{2}\left(1-t^{2}\right)}\right)}{1+\sqrt{1+r^{2}\left(1-t^{2}\right)}} & \text { if } g=0 .\end{cases}
$$

Thus the equation of $\Pi_{-1}(\mathcal{C})$ is 


$$
\left(s+\frac{1}{r \sqrt{1+\tanh ^{2} g}-\tanh g}\right)^{2}+t^{2}=\frac{r^{2}\left(1+\tanh ^{2} g\right)-\tanh ^{2} g+1}{\left(r \sqrt{1+\tanh ^{2} g}-\tanh g\right)^{2}} .
$$

This means that $\Pi_{-1}(\mathcal{C})$ is a sphere; hence, because $\Pi_{-1}\left(\tilde{\mathcal{K}}_{-1}^{n}\right)$ is the Poincaré model, $\mathcal{C}$ belongs to an isodistant, so the lemma is proved.

Let $p \in \mathbb{R}$ be such that $p \boldsymbol{b}_{n+1} \in \mathcal{P}\left(\frac{w-\tau_{\kappa}(g) \boldsymbol{b}_{n+1}}{\sqrt{1+\tau_{\kappa}^{2}(g)}}, \frac{\sigma_{\kappa}(\rho)}{\sqrt{\eta_{\kappa}^{2}(g)+\sigma_{\kappa}^{2}(g)}}\right)$ if $g>0$, and let $p$ be $\infty$ if $g=0$. Then (3.1) and (3.2) immediately give that

$$
p=\left\{\begin{array}{ll}
-\sigma_{\kappa}(\varrho) / \sigma_{\kappa}(g) & \text { if } g>0, \\
-\operatorname{sign}(\varrho) \infty & \text { if } g=0,
\end{array} \quad \text { and } \quad r=\frac{\left|\sigma_{\kappa}(\varrho)\right|}{\sqrt{\eta_{\kappa}^{2}(g)+\sigma_{\kappa}^{2}(g)}} .\right.
$$

It is worth noting that $g>0$ if and only if $p \in \mathbb{R}, g=0$ if and only if $p= \pm \infty$, and $p=0$ if $\varrho=0$ and $g>0$.

\section{The slice transform}

Lemma 3.1 gives rise to consider the slice transform $\mathrm{S}^{\kappa}$. We call the intersections of $\mathcal{K}_{\kappa}^{n}$ with hyperplanes slices. The slice transform $S^{\kappa}$ sends every suitable (not necessarily even) function $h$ on $\mathcal{K}_{\kappa}^{n}$ to the function $S^{\kappa} h$ on the set of slices so that $S^{\kappa} h$ gives for every slice the integral of $h$ over that slice.

To determine $S^{\kappa}$, firstly we define some special slice transforms $S_{ \pm}^{\kappa}$, for which we need the "inverses" $\Pi_{p}^{\kappa ; \pm}$ of the mappings $\Pi_{p}$ from $\Pi_{p}\left(\mathcal{K}_{\kappa}^{n}\right)$ into $\mathcal{K}_{\kappa}^{n}$.

Define the embedding $\Gamma: \mathbb{R}^{n} \rightarrow \mathcal{A}_{0}^{n} \subset \mathbb{R}^{n+1}$ by $\Gamma\left(x_{1}, \ldots, x_{n}\right)=\left(x_{1}, \ldots, x_{n}, 0\right)$. Then it is easy to see that

$$
\overline{\mathcal{M}}_{\kappa ; p+1}^{n}:=\Pi_{p}\left(\mathcal{K}_{\kappa}^{n}\right)=(p+1) \boldsymbol{b}_{n+1}+\Gamma\left(\mathcal{M}_{\kappa ; p}^{n}\right),
$$

where

$$
\mathcal{M}_{\kappa ; p}^{n}= \begin{cases}\frac{1}{\sqrt{1-p^{2}}} \mathcal{B}^{n}, & \text { if } \kappa=-1 \text { and }|p|<1, \\ \mathbb{R}^{n}, & \text { if } \kappa=-1 \text { and }|p| \geq 1 \text { or } p= \pm \infty, \\ \mathbb{R}^{n}, & \text { if } \kappa=0 \text { and } p \in \mathbb{R} \text { or } p= \pm \infty, \\ \mathbb{R}^{n}, & \text { if } \kappa=1 \text { and }|p| \leq 1, \\ \frac{1}{\sqrt{p^{2}-1}} \mathcal{B}^{n}, & \text { if } \kappa=1 \text { and }|p|>1, \\ \mathcal{B}^{n}, & \text { if } \kappa=1 \text { and } p= \pm \infty\end{cases}
$$

From now on

$$
\text { we do not differentiate between the vectors corresponding through } \Gamma \text {. }
$$

Fix a unit vector $\boldsymbol{u} \in \mathcal{S}^{n} \cap \mathcal{A}_{0}^{n}$. Then every point of $\overline{\mathcal{M}}_{\kappa ; p+1}^{n}$ can be uniquely ( $p \boldsymbol{b}_{n+1}$ is an exception) written in the form $p \boldsymbol{b}_{n+1}+e \boldsymbol{u}$, where $e \in[0, \infty)$. So there are functions $\nu: \mathbb{R}_{+} \rightarrow \mathbb{R}$ such that the point $p \boldsymbol{b}_{n+1}+v(e)\left(\boldsymbol{b}_{n+1}+e \boldsymbol{u}\right)$ is in $\mathcal{K}_{\kappa}^{n}$. (See Fig. 2.) 

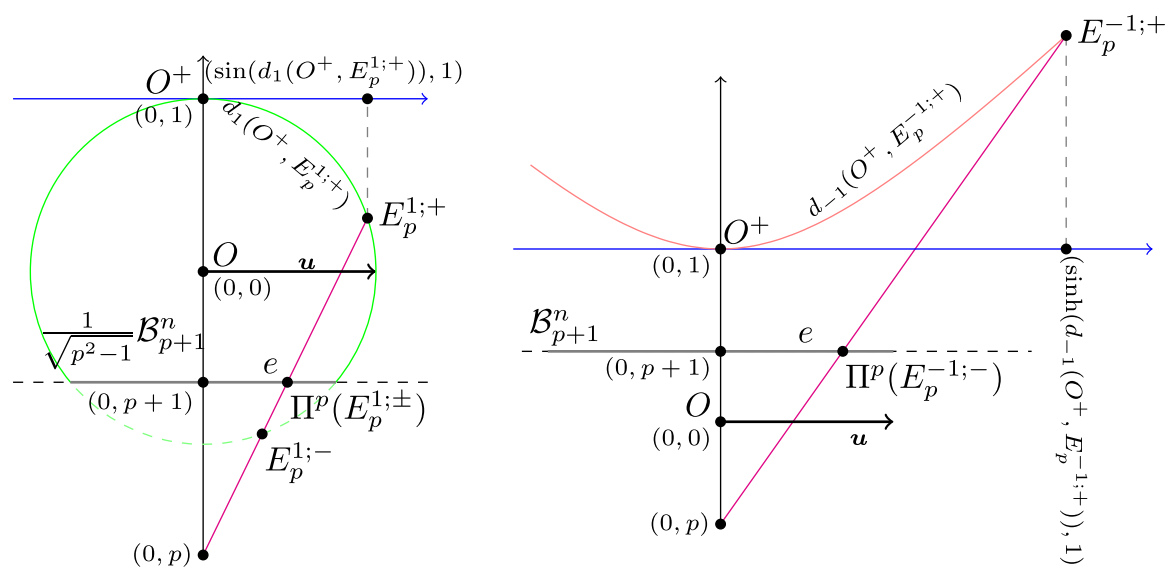

Fig. 2 Depiction of the plane spanned by $\boldsymbol{u}$ and $\boldsymbol{b}_{n+1}$ shows the case $g>0$, i.e., $p=-\sigma_{\kappa}(\varrho) / \sigma_{\kappa}(g)$, for spaces of $\kappa=1$ and $\kappa=-1$

Thus $\kappa v^{2}(e) e^{2}+(p+v(e))^{2}=1$, hence we obtain

$$
\nu_{p}^{\kappa ; \pm}(e)=\frac{-p \pm \sqrt{1-\kappa e^{2}\left(p^{2}-1\right)}}{1+\kappa e^{2}} .
$$

This and (4.1) allow us to define the mapping

$$
\Pi_{p}^{\kappa ; \pm}: \overline{\mathcal{M}}_{\kappa ; p+1}^{n} \ni x \boldsymbol{u}+(p+1) \boldsymbol{b}_{n+1} \mapsto p \boldsymbol{b}_{n+1}+v_{p}^{\kappa ; \pm}(x)\left(\boldsymbol{b}_{n+1}+x \boldsymbol{u}\right) \in \mathcal{K}_{\kappa}^{n} .
$$

Observe that $v_{ \pm 1}^{\kappa ; \pm}$ vanishes, so $\Pi_{ \pm 1}^{\kappa ; \pm}\left(x \boldsymbol{v}+( \pm 1+1) \boldsymbol{b}_{n+1}\right)= \pm \boldsymbol{b}_{n+1}$. Further, the mapping $\Pi_{p}^{-1 ;+}$ is the inverse of $\Pi_{p} \uparrow_{\hat{\mathcal{K}}_{-1}^{n}}$ if $p \leq 0$, the mapping $\Pi_{p}^{0 ;+}$ is the inverse of $\Pi_{p} \uparrow_{\hat{\mathcal{K}}_{0}^{n}}$ if $p \in \mathbb{R} \backslash\{1\}$, but the mapping $\Pi_{p}^{1 ; \pm}$ is the inverse of $\Pi_{p} \uparrow_{\mathcal{K}_{1}^{n}}$ if and only if $p=\mp 1$.

We define the special slice transforms $S_{ \pm}^{\kappa}$ for suitable functions $h$ in $C\left(\mathcal{K}_{\kappa}^{n}\right)$ by

$$
\mathrm{S}_{ \pm}^{\kappa} h(p ; \boldsymbol{w}, q)=\int_{\mathcal{S}_{w, q}^{n-1}} h\left(\Pi_{p}^{\kappa ; \pm}\left(e_{q}(\langle\boldsymbol{w}, \boldsymbol{u}\rangle) \boldsymbol{u}+(p+1) \boldsymbol{b}_{n+1}\right)\right) \omega_{p ; q}^{\kappa ; \pm}(\boldsymbol{w}, \boldsymbol{u}) \mathrm{d} \boldsymbol{u},
$$

where $\quad p \in \mathbb{R}, \quad q>0, \quad w \in \mathcal{S}^{n-1}, \quad e_{q}(x)=q / x \quad$ for $\quad x \in(0,1] \quad$ (recall (2.1)), $\mathcal{S}_{\boldsymbol{w}, q}^{n-1}=\left\{\boldsymbol{u} \in \mathcal{S}^{n-1}: e_{q}(\langle\boldsymbol{u}, \boldsymbol{w}\rangle) \boldsymbol{u} \in \mathcal{M}_{\kappa ; p}^{n}\right\}, d \boldsymbol{u}$ is the standard surface measure of $\mathcal{S}^{n-1}$, and $\omega_{p ; q}^{\kappa ; \pm}$ is the density pulled back by $\Pi_{p}$ from the hypersurface $\mathcal{K}_{\kappa}^{n} \cap \mathcal{P}\left(\frac{w-q b_{n+1}}{\sqrt{1+q^{2}}}, \frac{q|p|}{\sqrt{1+q^{2}}}\right)$. with metric $g_{\kappa}$.

Theorem 4.1 With $e=e_{q}(\langle\boldsymbol{w}, \boldsymbol{u}\rangle)$ we have

$$
\omega_{p ; q}^{\kappa ; \pm}(\boldsymbol{w}, \boldsymbol{u})=\frac{\sqrt{1+\kappa q^{2}\left(1-p^{2}\right)}}{q} \frac{\left|\nu_{p}^{\kappa ; \pm}(e)\right|^{n-1} e^{n}}{\sqrt{1-\kappa e^{2}\left(p^{2}-1\right)}} .
$$

Proof Firstly, assume $n=2$ and let $\boldsymbol{u}_{\varphi}=(\cos \varphi, \sin \varphi, 0)$. Then the isodistant's point in the direction $\boldsymbol{u}_{\varphi}$ is $E_{p}^{\kappa ; \pm}\left(e_{q}(\cos \varphi) \boldsymbol{u}_{\varphi}\right)=\prod_{p}^{\kappa ; \pm}\left(e_{q}(\cos \varphi) \boldsymbol{u}_{\varphi}+(p+1) \boldsymbol{b}_{n+1}\right)$, so (4.4) gives 


$$
E_{p}^{\kappa ; \pm}\left(e_{q}(\cos \varphi) \boldsymbol{u}_{\varphi}\right)=p \boldsymbol{b}_{n+1}+v_{p}^{\kappa ; \pm}\left(e_{q}(\cos \varphi)\right)\left(\boldsymbol{b}_{n+1}+e_{q}(\cos \varphi) \boldsymbol{u}_{\varphi}\right),
$$

where $v_{p}^{\kappa ; \pm}$ is given by (4.3). Since $\boldsymbol{u}_{\varphi}=\left(\frac{q}{e_{q}(\cos \varphi)}, \sqrt{1-\frac{q^{2}}{e_{q}^{2}(\cos \varphi)}}, 0\right)$, this gives

$$
\begin{aligned}
& E_{p}^{\kappa ; \pm}\left(e_{q}(\cos \varphi) \boldsymbol{u}_{\varphi}\right) \\
& =\left(v_{p}^{\kappa ; \pm}\left(e_{q}(\cos \varphi)\right) q, v_{p}^{\kappa ; \pm}\left(e_{q}(\cos \varphi)\right) \sqrt{e_{q}^{2}(\cos \varphi)-q^{2}}, p+v_{p}^{\kappa ; \pm}\left(e_{q}(\cos \varphi)\right)\right) .
\end{aligned}
$$

This shows that $E_{p}^{\kappa ; \pm}$ depends only on $e_{q}$, hence we can take

$$
\frac{\mathrm{d} E_{p}^{\kappa ; \pm}}{\mathrm{d} e}=\left(\dot{v}_{p}^{\kappa ; \pm}(e) q, \dot{v}_{p}^{\kappa ; \pm}(e) \sqrt{e^{2}-q^{2}}+v_{p}^{\kappa ; \pm}(e) \frac{e}{\sqrt{e^{2}-q^{2}}}, \dot{v}_{p}^{\kappa ; \pm}(e)\right),
$$

so we obtain

$$
\omega_{p ; q}^{\kappa ; \pm}(\boldsymbol{u})=\left|\frac{\mathrm{d} E_{p}^{\kappa ; \pm}}{\mathrm{d} e}\left(e_{q}(\langle\boldsymbol{w}, \boldsymbol{u}\rangle)\right)\right|_{\kappa}\left|\frac{\mathrm{d}\left(e_{q} \circ \cos \right)}{\mathrm{d} \varphi}(\arccos (\langle\boldsymbol{w}, \boldsymbol{u}\rangle))\right| .
$$

Here $\frac{\mathrm{d}\left(e_{q} \circ \cos \right)}{\mathrm{d} \varphi}=\frac{q \sin \varphi}{\cos ^{2} \varphi}$, and, from (4.8) with respect to (1.2), we get

$$
\left|\frac{\mathrm{d} E_{p}^{\kappa ; \pm}}{\mathrm{d} e}\right|_{\kappa}^{2}=\left(\dot{\nu}_{p}^{\kappa ; \pm}(e)\right)^{2} q^{2}+\frac{\left(\dot{\nu}_{p}^{\kappa ; \pm}(e)\left(e^{2}-q^{2}\right)+v_{p}^{\kappa ; \pm}(e) e\right)^{2}}{e^{2}-q^{2}}+\kappa\left(\dot{\nu}_{p}^{\kappa ; \pm}(e)\right)^{2} .
$$

From (4.3) we obtain

$$
\begin{aligned}
\dot{\nu}_{p}^{\kappa ; \pm}(e) & =\frac{ \pm \frac{-\kappa e\left(p^{2}-1\right)}{\sqrt{1-\kappa e^{2}\left(p^{2}-1\right)}}}{1+\kappa e^{2}}-\frac{\left(-p \pm \sqrt{1-\kappa e^{2}\left(p^{2}-1\right)}\right) 2 \kappa e}{\left(1+\kappa e^{2}\right)^{2}}=\frac{\mp \kappa e\left(\nu_{p}^{\kappa ; \pm}(e)\right)^{2}}{\sqrt{1-\kappa e^{2}\left(p^{2}-1\right)}} \\
& =\frac{-\kappa e\left(\nu_{p}^{\kappa ; \pm}(e)\right)^{2}}{p+\left(1+\kappa e^{2}\right) \nu_{p}^{\kappa ; \pm}(e)}
\end{aligned}
$$

which, by substitution into (4.9), leads to

$$
\begin{aligned}
& \left|\frac{\mathrm{d} E_{p}^{\kappa ; \pm}}{\mathrm{d} e}\right|_{\kappa}^{2} \\
& =\frac{e^{2}\left(\nu_{p}^{\kappa ; \pm}(e)\right)^{2}}{\left(p+\left(1+\kappa e^{2}\right) v_{p}^{\kappa ; \pm}(e)\right)^{2}} \\
& \times\left(\kappa\left(1+\kappa q^{2}\right)\left(v_{p}^{\kappa ; \pm}(e)\right)^{2}+\frac{\left(p+\left(1+\kappa e^{2}\right) v_{p}^{\kappa ; \pm}(e)-\kappa\left(e^{2}-q^{2}\right) v_{p}^{\kappa ; \pm}(e)\right)^{2}}{e^{2}-e^{2}}\right) \\
& =\frac{e^{2}\left(\nu_{p}^{\kappa ; \pm}(e)\right)^{2}}{1-\kappa e^{2}\left(p^{2}-1\right)}\left(\kappa\left(1+\kappa q^{2}\right)\left(v_{p}^{\kappa ; \pm}(e)\right)^{2}+\frac{\left(p+\left(1+\kappa q^{2}\right) \nu_{p}^{\kappa ; \pm}(e)\right)^{2}}{e^{2}-q^{2}}\right) .
\end{aligned}
$$

So letting $e=e_{q}(\langle\boldsymbol{w}, \boldsymbol{u}\rangle)$, we conclude that 


$$
\omega_{p ; q}^{\kappa ; \pm}(\boldsymbol{u})=\left|v_{p}^{\kappa ; \pm}(e)\right| e^{2} \frac{\sqrt{\kappa\left(1+\kappa q^{2}\right)\left(v_{p}^{\kappa ; \pm}(e)\right)^{2}\left(e^{2}-q^{2}\right)+\left(p+\left(1+\kappa q^{2}\right) \nu_{p}^{\kappa ; \pm}(e)\right)^{2}}}{q \sqrt{1-\kappa e^{2}\left(p^{2}-1\right)}},
$$

where the expression ( $\ddagger)$ under the square root sign can be simplified as follows:

$$
\begin{aligned}
\ddagger & =\kappa\left(1+\kappa q^{2}\right)\left(v_{p}^{\kappa ; \pm}(e)\right)^{2}\left(e^{2}-q^{2}\right)+\left(p+\left(1+\kappa q^{2}\right) v_{p}^{\kappa ; \pm}(e)\right)^{2} \\
& =\left(1+\kappa q^{2}\right)\left(v_{p}^{\kappa ; \pm}(e)\right)^{2}\left(1+\kappa e^{2}\right)+p^{2}+2 p\left(1+\kappa q^{2}\right) v_{p}^{\kappa ; \pm}(e) \\
& =p^{2}+\frac{1+\kappa q^{2}}{1+\kappa e^{2}}\left(1+\kappa e^{2}\right) u_{p}^{\kappa ; \pm}(e)\left(u_{p}^{\kappa ; \pm}(e)\left(1+\kappa e^{2}\right)+2 p\right) \\
& =p^{2}+\frac{1+\kappa q^{2}}{1+\kappa e^{2}}\left(-p \pm \sqrt{1-\kappa e^{2}\left(p^{2}-1\right)}\right)\left(p \pm \sqrt{1-\kappa e^{2}\left(p^{2}-1\right)}\right) \\
& =p^{2}+\frac{1+\kappa q^{2}}{1+\kappa e^{2}}\left(1-\kappa e^{2}\left(p^{2}-1\right)-p^{2}\right)=p^{2}+\left(1+\kappa q^{2}\right)\left(1-p^{2}\right) \\
& =1+\kappa q^{2}\left(1-p^{2}\right) .
\end{aligned}
$$

To get $\omega_{p ; q}^{\kappa ; \pm}$ for higher dimension $n$, we only have to multiply its 2-dimensional version with $\sigma_{\kappa}^{n-2}\left(d_{\kappa}\left(O^{+}, E_{p}^{\kappa ; \pm}\left(e_{q}(\langle\boldsymbol{w}, \boldsymbol{u}\rangle)\right)\right)\right)$, because $\mathcal{K}_{\kappa}^{n}$ is the rotational manifold with size function $\sigma_{\kappa}$. As $\sigma_{\kappa}\left(d_{\kappa}\left(O^{+}, E_{p}^{\kappa ; \pm}\left(e_{q}\right)\right)\right)=\left|\left\langle\boldsymbol{u}_{\varphi}, E_{p}^{\kappa ; \pm}\left(e_{q}\right)\right\rangle\right|=\left|\nu_{p}^{\kappa ; \pm}\left(e_{q}\right)\right| e_{q}$, we arrive at (4.6).

Fix a unit vector $\boldsymbol{u} \in \mathcal{S}^{n} \cap \mathcal{A}_{0}^{n}$. Then every point of $\Pi_{\infty}\left(\mathcal{K}_{\kappa}^{n}\right)$ in the plane spanned by $\boldsymbol{u}$ and $\boldsymbol{b}_{n+1}$ can be uniquely written in the form $\boldsymbol{\infty} \boldsymbol{b}_{n+1}+e \boldsymbol{u}$, where $e \in \mathbb{R}$. So there are functions $v: \mathbb{R}_{+} \rightarrow \mathbb{R}$ such that the point $v(e) \boldsymbol{b}_{n+1}+v(e) \boldsymbol{u}$ is in $\mathcal{K}_{\kappa}^{n}$. (See Fig. 3.)

Thus $\kappa v^{2}(e)+v^{2}(e)=1$, hence we obtain $v_{ \pm \infty}^{\kappa ; \pm}(e)= \pm \sqrt{1-\kappa e^{2}}$. This and (4.1) allow us to define the mapping

$$
\Pi_{\infty}^{\kappa ; \pm}: \overline{\mathcal{M}}_{\kappa ; \infty}^{n} \ni x \boldsymbol{v}+\infty \boldsymbol{b}_{n+1} \mapsto \pm \sqrt{1-\kappa x^{2}} \boldsymbol{b}_{n+1}+x \boldsymbol{v} \in \mathcal{K}_{\kappa}^{n}
$$
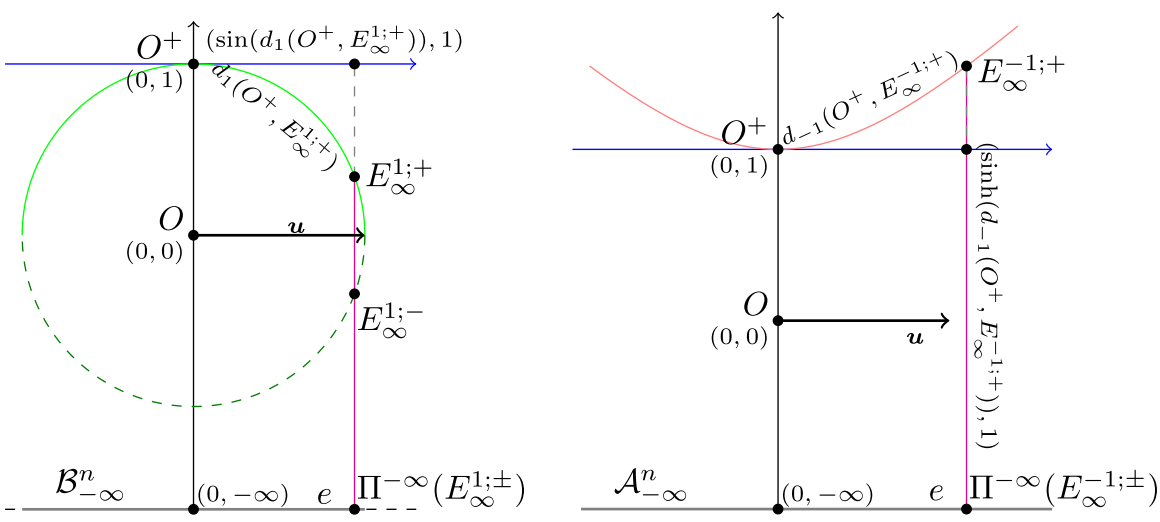

Fig. 3 Depiction of the plane spanned by $\boldsymbol{u}$ and $\boldsymbol{b}_{n+1}$ shows the case $g=0$, i.e., $p=-\operatorname{sign}(\varrho) \infty$, for the spaces of $\kappa=1$ and $\kappa=-1$ 
We define the special slice transforms $S_{ \pm}^{\kappa}$ for suitable functions $h$ in $C\left(\mathcal{K}_{\kappa}^{n}\right)$ by

$$
\mathrm{S}_{ \pm}^{\kappa} h(\infty ; \boldsymbol{w}, q)=\int_{\mathcal{S}_{w, q}^{n-1}} h\left(\Pi_{\infty}^{\kappa ; \pm}\left(e_{q}(\langle\boldsymbol{w}, \boldsymbol{u}\rangle) \boldsymbol{u}+\infty \boldsymbol{b}_{n+1}\right)\right) \omega_{\infty ; q}^{\kappa ; \pm}(\boldsymbol{w}, \boldsymbol{u}) \mathrm{d} \boldsymbol{u},
$$

where $q>0, \boldsymbol{w} \in \mathcal{S}^{n-1}, e_{q}(x)=q / x$ for $x \in(0,1]$ (recall $\left.(2.1)\right), \mathcal{S}_{w, q}^{n-1}=\left\{\boldsymbol{u} \in \mathcal{S}^{n} \cap \mathcal{A}_{0}^{n}\right.$ : $\left.e_{q}(\langle\boldsymbol{u}, \boldsymbol{w}\rangle) \boldsymbol{u} \in \mathcal{M}_{\kappa ; \infty}^{n}\right\}, d \boldsymbol{u}$ is the standard surface measure on $\mathcal{S}^{n} \cap \mathcal{A}_{0}^{n}$, and $\omega_{\infty ; q}^{\kappa ; \pm}$ is the density pulled back by $\Pi_{\infty}$ from the hypersurface $\mathcal{K}_{\kappa ; \infty ; \pm}^{n} \cap \mathcal{P}(\boldsymbol{w}, q)$.

Theorem 4.2 We have

$$
\omega_{\infty ; q}^{\kappa ; \pm}(\boldsymbol{w}, \boldsymbol{u})=\frac{e_{q}^{n}(\langle\boldsymbol{w}, \boldsymbol{u}\rangle) \sqrt{1-\kappa q^{2}}}{q \sqrt{1-\kappa e_{q}^{2}(\langle\boldsymbol{w}, \boldsymbol{u}\rangle)}}
$$

Proof Firstly, assume $n=2$ and let $\boldsymbol{u}_{\varphi}=(\cos \varphi, \sin \varphi, 0)$. Then the isodistant's point in the direction $\boldsymbol{u}_{\varphi}$ is $E_{p}^{\kappa ; \pm}\left(e_{q}(\cos \varphi) \boldsymbol{u}_{\varphi}\right)=\prod_{\infty}^{\kappa ; \pm}\left(e_{q}(\cos \varphi) \boldsymbol{u}_{\varphi}+\infty \boldsymbol{b}_{n+1}\right)$, so (4.10) gives

$$
E_{\infty}^{\kappa ; \pm}\left(e_{q}(\cos \varphi) \boldsymbol{u}_{\varphi}\right)=e_{q}(\cos \varphi) \boldsymbol{u}_{\varphi} \pm \sqrt{1-\kappa e_{q}^{2}(\cos \varphi)} \boldsymbol{b}_{n+1} .
$$

Since $\boldsymbol{u}_{\varphi}=\left(\frac{q}{e_{q}(\cos \varphi)}, \sqrt{1-\frac{q^{2}}{e_{q}^{2}(\cos \varphi)}}, 0\right)$, we get from (4.13) that

$$
E_{\infty}^{\kappa ; \pm}\left(e_{q}(\cos \varphi) \boldsymbol{u}_{\varphi}\right)=\left(q, \sqrt{e_{q}^{2}(\cos \varphi)-q^{2}}, \pm \sqrt{1-\kappa e_{q}^{2}(\cos \varphi)}\right) .
$$

This shows that $E_{\infty}^{\kappa ; \pm}$ depends only on $e_{q}$, hence we can take

$$
\frac{\mathrm{d} E_{\infty}^{\kappa ; \pm}}{\mathrm{d} e}=\left(0, \frac{e}{\sqrt{e^{2}-q^{2}}}, \frac{\mp \kappa e}{\sqrt{1-\kappa e^{2}}}\right),
$$

and obtain

$$
\omega_{\infty ; q}^{\kappa ; \pm}(\boldsymbol{u})=\left|\frac{\mathrm{d} E_{\infty}^{\kappa ; \pm}}{\mathrm{d} e}\left(e_{q}(\langle\boldsymbol{w}, \boldsymbol{u}\rangle)\right)\right|_{\kappa}\left|\frac{\mathrm{d}\left(e_{q} \circ \cos \right)}{\mathrm{d} \varphi}(\arccos (\langle\boldsymbol{w}, \boldsymbol{u}\rangle))\right| .
$$

Here $\frac{\mathrm{d}\left(e_{q} \circ \cos \right)}{\mathrm{d} \varphi}=\frac{q \sin \varphi}{\cos ^{2} \varphi}$, and, by (1.2), we get

$$
\left|\frac{\mathrm{d} E_{\infty}^{\kappa ; \pm}}{\mathrm{d} e}\right|_{\kappa}^{2}=\frac{e^{2}}{e^{2}-q^{2}}+\kappa \frac{e^{2}}{1-\kappa e^{2}}=\frac{e^{2}\left(1-\kappa q^{2}\right)}{\left(e^{2}-q^{2}\right)\left(1-\kappa e^{2}\right)} .
$$

So letting $e=e_{q}(\langle\boldsymbol{w}, \boldsymbol{u}\rangle)$, we conclude that

$$
\omega_{\infty ; q}^{\kappa ; \pm}(\boldsymbol{u})=e^{2} \frac{\sqrt{1-\kappa q^{2}}}{q \sqrt{1-\kappa e^{2}}} .
$$

To get $\omega_{\infty ; q}^{\kappa ; \pm}$ for higher dimension $n$, we only have to multiply the 2-dimensional version with $\sigma_{\kappa}^{n-2}\left(d_{\kappa}\left(O^{+}, E_{\infty}^{\kappa ; \pm}\left(e_{q}(\langle\boldsymbol{w}, \boldsymbol{u}\rangle)\right)\right)\right)$, because $\mathcal{K}_{\kappa}^{n}$ is a rotational manifold. Since $\sigma_{\kappa}\left(d_{\kappa}\left(O^{+}, E_{\infty}^{\kappa ; \pm}\right)\right)=\left|\left\langle\boldsymbol{u}_{\varphi}, E_{\infty}^{\kappa ; \pm}\left(e_{q}(\cos \varphi)\right)\right\rangle\right|=e_{q}(\cos \varphi)$, we arrive at (4.12). 
To make their later use easier, we extend definitions (4.5) and (4.11) of the special slice transforms $\mathrm{S}_{ \pm}^{\kappa}$ by setting $\mathrm{S}_{ \pm}^{\kappa} h(p ; \boldsymbol{w}, q):=0$ for $p \in \mathbb{R}$ and $q>0$ if the hyperplane

$$
\begin{aligned}
& \mathcal{P}\left(\frac{\boldsymbol{w}-q \boldsymbol{b}_{n+1}}{\sqrt{1+q^{2}}}, \frac{q|p|}{\sqrt{1+q^{2}}}\right) \\
& \quad=\operatorname{span}\left[p \boldsymbol{b}_{n+1} ;(p+1) \boldsymbol{b}_{n+1}+\left\{\frac{q}{\langle\boldsymbol{u}, \boldsymbol{w}\rangle} \boldsymbol{u}: \boldsymbol{u} \in \mathcal{S}^{n-1},\langle\boldsymbol{u}, \boldsymbol{w}\rangle>0\right\}\right]
\end{aligned}
$$

does not intersect $\mathcal{K}_{\kappa}^{n}$, and by setting $\mathrm{S}_{ \pm}^{\kappa} h(\infty ; \boldsymbol{w}, q):=0$ for $q>0$ if the hyperplane $\mathcal{P}(\boldsymbol{w}, q)$ does not intersect $\mathcal{K}_{\kappa}^{n}$. With this understanding, the slice transform is

$$
\mathrm{S}^{\kappa} h(p ; \boldsymbol{w}, q)= \begin{cases}\mathrm{S}_{+}^{\kappa} h(p ; \boldsymbol{w}, q)+\mathrm{S}_{-}^{\kappa} h(p ; \boldsymbol{w}, q) & \text { if }|p| \neq 1, \\ \mathrm{~S}_{+}^{-1} h(p ; \boldsymbol{w}, q)+\mathrm{S}_{-}^{-1} h(p ; \boldsymbol{w}, q) & \text { if } p= \pm 1 \text { and } \kappa=-1, \\ \mathrm{~S}_{\mp}^{0} h(p ; \boldsymbol{w}, q)+\mathrm{S}_{ \pm}^{0} h(-p ; \boldsymbol{w}, 0) & \text { if } p= \pm 1 \text { and } \kappa=0, \\ \mathrm{~S}_{\mp}^{1} h(p ; \boldsymbol{w}, q) & \text { if } p= \pm 1 \text { and } \kappa=1,\end{cases}
$$

where $p \in \mathbb{R} \cup\{ \pm \infty\}, \boldsymbol{w} \in \mathcal{S}^{n-1}$, and $q \geq 0$.

\section{Intertwining relations between the slice transforms}

Following (4.1), we define the mappings $\Psi_{p}^{\kappa ; \pm}: \mathcal{M}_{\kappa ; p}^{n} \rightarrow \mathcal{K}_{\kappa}^{n}$ by

$$
\Psi_{p}^{\kappa ; \pm}(\boldsymbol{x})= \begin{cases}\Pi_{p}^{\kappa ; \pm}\left(\boldsymbol{x}+(p+1) \boldsymbol{b}_{n+1}\right) & \text { if } p \in \mathbb{R} \\ \Pi_{\infty}^{\kappa ; \pm}\left(\boldsymbol{x}+\infty \boldsymbol{b}_{n+1}\right) & \text { if } p=\infty\end{cases}
$$

where $\Pi_{p}^{\kappa ; \pm}$ and $\Pi_{\infty}^{\kappa ; \pm}$ are given by (4.4) and (4.10), respectively. Further, let $\bar{\Psi}_{p}^{\kappa ; \pm}$ be the inverse of $\Psi_{p}^{\kappa ; \pm}$, and define the spaces

$$
\mathcal{K}_{p}^{\kappa ; \pm}:=\operatorname{Im} \Psi_{p}^{\kappa ; \pm}, \text { and } \mathcal{K}_{\infty}^{\kappa ; \pm}:=\operatorname{Im} \Psi_{\infty}^{\kappa ; \pm} .
$$

Define also the operators $\mathrm{N}_{p}^{\kappa ; \pm}: C\left(\mathcal{M}_{\kappa ; p}^{n}\right) \ni f \mapsto \mathrm{N}_{p}^{\kappa ; \pm} f$ so that

$$
\mathrm{N}_{p}^{\kappa ; \pm} f: \mathcal{M}_{\kappa ; p}^{n} \ni \boldsymbol{x} \mapsto \mathrm{N}_{p}^{\kappa ; \pm} f(\boldsymbol{x})= \begin{cases}\frac{f(\boldsymbol{x})\left|\nu_{p}^{\kappa ; \pm}(|x|)\right|^{n-1}}{\sqrt{1-\kappa x^{2}\left(p^{2}-1\right)}} & \text { if } p \in \mathbb{R}, \\ \frac{f(\boldsymbol{x})}{\sqrt{1-\kappa x^{2}}} & \text { if } p=\infty,\end{cases}
$$

where $v_{p}^{\kappa ; \pm}$ is given by (4.3), and let $\overline{\mathrm{N}}_{p}^{\kappa ; \pm}$ be the inverse of $\mathrm{N}_{p}^{\kappa ; \pm}$.

We use the classical Euclidean Radon transform R (recall (2.2)) to formulate the following intertwining relations that are generalizations of [24, Theorem 2.1].

Theorem 5.1 Let $\kappa \in\{0, \pm 1\}, p \in \mathbb{R}$, and $f \in C\left(\mathcal{M}_{\kappa ; p}^{n}\right)$ be such that $\mathrm{Rf}$ exists. Define $h^{ \pm}: \mathcal{K}_{p}^{\kappa ; \pm} \rightarrow \mathbb{R}$ by $h^{ \pm} \circ \Psi_{p}^{\kappa ; \pm}=\overline{\mathrm{N}}_{p}^{\kappa ; \pm} f$. Then, for $q \neq 0$, we have

$$
\mathrm{S}_{ \pm}^{\kappa} h^{ \pm}(p ; \boldsymbol{w}, q)=\sqrt{1+\kappa q^{2}\left(1-p^{2}\right)} \operatorname{R} f(\boldsymbol{w}, q) .
$$


Proof By (4.5) we have

$$
\mathrm{S}_{ \pm}^{\kappa} h^{ \pm}(p ; \boldsymbol{w}, q)=\int_{\mathcal{S}_{w, 0}^{n-1}} f(e(\boldsymbol{u}) \boldsymbol{u}) \frac{\sqrt{1-\kappa e^{2}(\boldsymbol{u})\left(p^{2}-1\right)}}{\left|v_{p}^{\kappa ; \pm}(e(\boldsymbol{u}))\right|^{n-1}} \omega_{p ; q}^{\kappa ; \pm}(\boldsymbol{w}, \boldsymbol{u}) \mathrm{d} \boldsymbol{u},
$$

where $e(\boldsymbol{u})=e_{q}(\langle\boldsymbol{w}, \boldsymbol{u}\rangle)=\frac{q}{\langle\boldsymbol{w}, \boldsymbol{u}\rangle}$, and $\omega_{p ; q}^{K ; \pm}$ is given by (4.6). Substitution of (4.6) results in

$$
\mathrm{S}_{ \pm}^{\kappa} h^{ \pm}(p ; \boldsymbol{w}, q)=\frac{\sqrt{1+\kappa q^{2}\left(1-p^{2}\right)}}{q} \int_{\mathcal{S}_{w, 0}^{n-1}} f(e(\boldsymbol{u}) \boldsymbol{u}) e^{n}(\boldsymbol{u}) \mathrm{d} \boldsymbol{u},
$$

which proves the theorem by (2.2).

Theorem 5.2 Let $\kappa \in\{0, \pm 1\}$ and $f \in C\left(\mathcal{M}_{\kappa ; \infty}^{n}\right)$ be such that $\mathrm{R} f$ exists. Define $h^{ \pm}: \mathcal{K}_{p}^{\kappa ; \pm} \rightarrow \mathbb{R}$ by $h^{ \pm} \circ \Psi_{\infty}^{\kappa ; \pm}=\overline{\mathrm{N}}_{\infty}^{\kappa ; \pm} f$. Then for $q \neq 0$ we have

$$
\mathrm{S}_{ \pm}^{\kappa} h^{ \pm}(\infty ; \boldsymbol{w}, q)=\sqrt{1-\kappa q^{2}} \mathrm{R} f(\boldsymbol{w}, q) .
$$

Proof By (4.11) we have

$$
\mathrm{S}_{ \pm}^{\kappa} h^{ \pm}(\infty ; \boldsymbol{w}, q)=\int_{\mathcal{S}_{w, 0}^{n-1}} f(e(\boldsymbol{u}) \boldsymbol{u}) \sqrt{1-\kappa e^{2}(\boldsymbol{u})} \omega_{\infty ; q}^{\kappa ; \pm}(\boldsymbol{u}) \mathrm{d} \boldsymbol{u},
$$

where $e(\boldsymbol{u})=e_{q}(\langle\boldsymbol{w}, \boldsymbol{u}\rangle)=\frac{q}{\langle\boldsymbol{w}, \boldsymbol{u}\rangle}$, and $\omega_{\infty ; q}^{\kappa ; \pm}$ is given by (4.12). Substitution of (4.12) results in

$$
\mathrm{S}_{ \pm}^{\kappa} h^{ \pm}(\infty ; \boldsymbol{w}, q)=\frac{\sqrt{1-\kappa q^{2}}}{q} \int_{\mathcal{S}_{w, 0}^{n-1}} f(e(\boldsymbol{u}) \boldsymbol{u}) e^{n}(\boldsymbol{u}) \mathrm{d} \boldsymbol{u},
$$

which proves the theorem by (2.2).

\section{Shifted Funk transforms: support theorems and kernels}

The $p$-shifted Funk transform ( $p \in \mathbb{R} \cup\{ \pm \infty\}$ ) of a suitable function $h$ on $\mathcal{K}_{\kappa}^{n}$ is

$$
\mathrm{F}_{p}^{\kappa} h: \mathcal{S}^{n-1} \times \mathbb{R}_{>0} \ni(\boldsymbol{w}, q) \mapsto \mathrm{S}^{\kappa} h(p ; \boldsymbol{w}, q) .
$$

The proofs of the following support theorems in this section follow the method used in the proof of [24, Theorem 3.2]: we pull Support Theorem 2.1 back to $\mathcal{K}_{\kappa}^{n}$ through the adequate intertwining relation of (5.4) and (5.5).

Following (4.2), let

$$
\ell_{p}=\left\{\begin{array}{ll}
\infty & \text { if } \kappa p^{2} \leq \kappa, \\
1 / \sqrt{\left|p^{2}-1\right|} & \text { if } \kappa p^{2}>\kappa,
\end{array}(p \in \mathbb{R}) \text { and } \ell_{\infty}= \begin{cases}1 & \text { if } \kappa=1, \\
\infty & \text { if } \kappa \leq 0\end{cases}\right.
$$

Then, since $\mathcal{K}_{\kappa}^{n}$ is a rotational manifold, we can define the nonnegative functions

$$
\delta_{p}^{\kappa ; \pm}:\left[0, \ell_{p}\right) \rightarrow \mathbb{R}_{+} \text {such that } \delta_{p}^{\kappa ; \pm}(|\boldsymbol{x}|)=d_{\kappa}\left(O^{ \pm}, \Psi_{p}^{\kappa ; \pm}(\boldsymbol{x})\right) .
$$


Since $\sigma_{\kappa}$ is the size function of $\mathcal{K}_{\kappa}^{n}$, and so we have

$$
\begin{aligned}
& \sigma_{\kappa}\left(d_{\kappa}\left(O^{ \pm}, \Psi_{p}^{\kappa ; \pm}(\boldsymbol{x})\right)= \begin{cases}v_{p}^{\kappa ; \pm}(|\boldsymbol{x}|)|\boldsymbol{x}| & \text { if } p \in \mathbb{R} \text { by }(4.7), \\
|\boldsymbol{x}| & \text { if } p=\infty \text { by (4.13), }\end{cases} \right. \\
& \eta_{\kappa}\left(d_{\kappa}\left(O^{ \pm}, \Psi_{p}^{\kappa ; \pm}(\boldsymbol{x})\right)= \begin{cases}p+v_{p}^{\kappa ; \pm}(|\boldsymbol{x}|) & \text { if } p \in \mathbb{R} \text { by (4.7), } \\
\sqrt{1-\kappa|\boldsymbol{x}|^{2}} & \text { if } p=\infty \text { by (4.13), }\end{cases} \right.
\end{aligned}
$$

we deduce that

$$
\sigma_{\kappa}\left(\delta_{p}^{\kappa ; \pm}(e)\right)=v_{p}^{\kappa ; \pm}(e) e, \eta_{\kappa}\left(\delta_{p}^{\kappa ; \pm}(e)\right)=p+v_{p}^{\kappa ; \pm}(e)
$$

for $p \in \mathbb{R}$, and

$$
\sigma_{\kappa}\left(\delta_{\infty}^{\kappa ; \pm}(e)\right)=e, \eta_{\kappa}\left(\delta_{\infty}^{\kappa ; \pm}(e)\right)=\sqrt{1-\kappa e^{2}}
$$

Substituting $v_{p}^{\kappa ; \pm}$ from (4.3) into (6.4), it is easy to see that $\delta_{p}^{\kappa ; \pm}$ is strictly monotone increasing for $\kappa \geq 0$, and if $\kappa=-1$, then it is strictly monotone increasing in $[0,1)$ and decreasing in $(1, \infty)$. It is clear from $(6.5)$ that $\delta_{\infty}^{\kappa ; \pm}$ is strictly monotone increasing.

Let $\mathcal{L} \subset \mathcal{K}_{\kappa}^{n}$ be a nonempty, open domain, and define the set $C_{m}\left(\mathcal{K}_{\kappa}^{n}, \mathcal{L}\right)(m \in \mathbb{N})$ of all continuous functions $h$ on $\mathcal{K}_{\kappa}^{n}$ that satisfy

$$
h(E)= \begin{cases}O(1) \sigma_{\kappa}^{-m}\left(d_{\kappa}(E, P)\right) & \text { if } d_{\kappa}(E, P) \rightarrow l_{\kappa}, \\ O(1) \sigma_{\kappa}^{-m}\left(d_{\kappa}(E, \partial \mathcal{L})\right) & \text { if } E \rightarrow \partial \mathcal{L},\end{cases}
$$

where $P \in \mathcal{K}_{\kappa}^{n}$ is any fixed point, and the usual big-O notation is in use. We use the abbreviations $C_{m}\left(\mathcal{K}_{\kappa}^{n}\right):=C_{m}\left(\mathcal{K}_{\kappa}^{n}, \emptyset\right)$, and $C_{m}\left(\mathcal{K}_{\kappa}^{n}, p\right):=C_{m}\left(\mathcal{K}_{\kappa}^{n}, \mathcal{K}_{p}^{\kappa ; \pm}\right)($ see $(5.2))$.

\subsection{Support theorems}

We start with the elliptic case.

Theorem 6.1 Support theorems for shifted Funk transform on the sphere $\mathcal{K}_{1}^{n}$.

$\langle\mathrm{se} 1\rangle$ If $h \in C_{\infty}\left(\mathcal{K}_{1}^{n}, \pm 1\right)$ and $\mathrm{F}_{ \pm 1}^{1} h(\boldsymbol{w}, q)=0$ for every $q>s>0$ and $\boldsymbol{w} \in \mathcal{S}^{n-1}$, then $h\left(\operatorname{Exp}_{O^{\mp}}(\right.$ eu $\left.)\right)$ vanishes for every $e>2 \arctan (s)$ and $\boldsymbol{u} \in \mathcal{S}^{n-1}$.

$\langle\mathrm{se} 2\rangle \quad$ If $|p|<1, h \in C_{\infty}\left(\mathcal{K}_{1}^{n}, p\right)$ vanishes on $\mathcal{K}_{p}^{1 ; \mp}$, and $\mathrm{F}_{p}^{1} h(\boldsymbol{w}, q)=0$ for every $q>s>0$ and $\boldsymbol{w} \in \mathcal{S}^{n-1}$, then $h\left(\operatorname{Exp}_{O^{ \pm}}(e \boldsymbol{u})\right)$ vanishes for every $e^{p}>\delta_{p}^{1 ; \pm}(s)$ and $\boldsymbol{u} \in \mathcal{S}^{n-1}$.

$\langle\mathrm{se} 3\rangle \quad$ If $|p|>1, h \in C\left(\mathcal{K}_{1}^{n}\right)$ vanishes on $\mathcal{K}_{p}^{1 ; \mp}$, and $\mathrm{F}_{p}^{1} h(w, q)=0$ for every $q>s>0$ and $\boldsymbol{w} \in \mathcal{S}^{n-1}$, then $h\left(\operatorname{Exp}_{O^{ \pm}}(e \boldsymbol{u})\right)$ vanishes for every $\mathrm{P}>\delta_{p}^{1 ; \pm}(s)$ and $\boldsymbol{u} \in \mathcal{S}^{n-1}$.

$\langle\mathrm{se} 4\rangle \quad$ If $h \in C\left(\mathcal{K}_{1}^{n}\right)$ vanishes on $\mathcal{K}_{\infty}^{1 ; \mp}$, and $\mathrm{F}_{\infty}^{1} h(\boldsymbol{w}, q)=0$ for every $q>s>0$ and $\boldsymbol{w} \in \mathcal{S}^{n-1}$, then $h\left(\operatorname{Exp}_{O^{ \pm}}(\right.$eu $\left.)\right)$vanishes for everye $>\arcsin (s)$ and $\boldsymbol{u} \in \mathcal{S}^{n-1}$.

Proof We prove the statements one after the other.

$\left\langle\right.$ se1〉 As $|p|=1$, (4.2) gives that $\mathcal{M}_{1 ; \pm 1}^{n}=\mathbb{R}^{n}$. By symmetry, it is enough to prove for $p=-1$. So, according to (6.1) and (4.15), we have $\mathrm{F}_{-1}^{1} h(\boldsymbol{w}, q)=\mathrm{S}_{+}^{1} h(-1 ; \boldsymbol{w}, q)$. Let $f=\mathrm{N}_{-1}^{1 ;+}\left(h \circ \Psi_{-1}^{1 ;+}\right)$. Then $f \in C_{\infty}\left(\mathbb{R}^{n}\right)$ as $h \in C_{\infty}\left(\mathcal{K}_{1}^{n},-1\right)$. Further, by (5.4), $f$ satisfies $\mathrm{S}_{+}^{1} h(-1 ; \boldsymbol{w}, q)=\operatorname{Rf}(\boldsymbol{w}, q)$, hence $\mathrm{R} f$ vanishes for $q>s>0$. So, by Support Theorem 2.1, $f$ vanishes for $|x|>s>0$. Thus $h$ vanishes for $\Psi_{-1}^{1 ;+}(\boldsymbol{x})$ if $|\boldsymbol{x}|>s$, so the statement follows from (6.3) and (6.4). 
$\left\langle\right.$ se2〉 As $|p|<1,(4.2)$ gives that $\mathcal{M}_{1 ; p}^{n}=\mathbb{R}^{n}$. By symmetry, it is enough to prove in the case when $h$ vanishes on $\mathcal{K}_{p}^{1 ;-}$. So, by (6.1) and (4.15), we have $\mathrm{F}_{p}^{1} h(\boldsymbol{w}, q)=\mathrm{S}_{+}^{1} h(p ; \boldsymbol{w}, q)$. Let $f=\mathrm{N}_{p}^{1 ;+}\left(h \circ \Psi_{p}^{1 ;+}\right)$. Then $f \in C_{\infty}\left(\mathbb{R}^{n}\right)$, as $h \in C_{\infty}\left(\mathcal{K}_{1}^{n}, p\right)$. Further, by (5.4), $f$ satisfies $S_{+}^{1} h(p ; \boldsymbol{w}, q)=\sqrt{1+q^{2}\left(1-p^{2}\right)} \mathrm{R} f(\boldsymbol{w}, q)$, hence $\mathrm{R} f$ vanishes for $q>s>0$. So, by Support Theorem 2.1, $f$ vanishes for $|\boldsymbol{x}|>s>0$. Thus $h$ vanishes for $\Psi_{p}^{1 ;+}(\boldsymbol{x})$ if $|\boldsymbol{x}|>s$, hence the statement follows from (6.3) and (6.4).

$\langle\operatorname{se} 3\rangle \quad$ As $|p|>1$, (4.2) gives that $\mathcal{M}_{1 ; p}^{n}=\frac{1}{\sqrt{p^{2}-1}} \mathcal{B}^{n}$. By symmetry, it is enough to prove under the assumption that $h$ vanishes on $\mathcal{K}_{p}^{1 ;-}$. So, according to (6.1) and (4.15), we have $\mathrm{F}_{p}^{1} h(\boldsymbol{w}, q)=\mathrm{S}_{+}^{1} h(p ; \boldsymbol{w}, q)$. Let $f=\mathrm{N}_{p}^{1 ;+}\left(h \circ \Psi_{p}^{1 ;+}\right)$. Then $f$ has compact support. Further, $f$ satisfies $S_{+}^{1} h(p ; w, q)=\sqrt{1+q^{2}\left(1-p^{2}\right)} \operatorname{R} f(\boldsymbol{w}, q)$ by (5.4), hence $\mathrm{R} f$ vanishes for $q>s>0$. So, by Support Theorem 2.1, $f$ vanishes for $|x|>s>0$. Thus $h$ vanishes for $\Psi_{p}^{1 ;+}(\boldsymbol{x})$ if $|\boldsymbol{x}|>s$, hence the statement follows from (6.3) and (6.4).

$\left\langle\right.$ se4〉 As $p= \pm \infty,(4.2)$ gives that $\mathcal{M}_{1 ; p}^{n}=\mathcal{B}^{n}$. By symmetry, it is enough to prove under the assumption that $h$ vanishes on $\mathcal{K}_{1}^{1 ;-}$. So, according to (6.1) and (4.15), we have $\mathrm{F}_{\infty}^{1} h(\boldsymbol{w}, q)=\mathrm{S}_{+}^{1} h(\infty ; \boldsymbol{w}, q)$. Let $f=\mathrm{N}_{\infty}^{1 ;+}\left(h \circ \Psi_{\infty}^{1 ;+}\right)$. Then $f$ has compact support. Further, $f$ satisfies $S_{+}^{1} h(\infty ; \boldsymbol{w}, q)=\sqrt{1-q^{2}} \mathrm{R} f(\boldsymbol{w}, q)$ by $(5.5)$, hence $\mathrm{R} f$ vanishes for $q>s>0$. So, by Support Theorem 2.1, $f$ vanishes for $|x|>s>0$. Thus $h$ vanishes for $\Psi_{\infty}^{1 ;+}(\boldsymbol{x})$ if $|\boldsymbol{x}|>s$, hence the statement follows from (6.3) and (6.4).

Statement $\langle$ se1 $\rangle$ is a slight sharpening of [18, Corollary 1.27 in Chapter III] about the spherical slice transform.

We continue with the Euclidean case.

Theorem 6.2 Support theorems for shifted Funk transforms on the hyperplane $\mathcal{K}_{0}^{n}$

$\langle\mathrm{sp} 1\rangle \quad$ If $h \in C_{\infty}\left(\mathcal{K}_{0}^{n}, \pm 1\right)$ and $\mathrm{F}_{ \pm 1}^{0} h(\boldsymbol{w}, q)=0$ for every $q>s>0$ and $\boldsymbol{w} \in \mathcal{S}^{n-1}$, then $h(e \boldsymbol{u})$ vanishes for every $e>2 s$ and $\boldsymbol{u} \in \mathcal{S}^{n-1}$.

$\langle\mathrm{sp} 2\rangle \quad$ If $|p| \neq 1, h \in C_{\infty}\left(\mathcal{K}_{0}^{n}, p\right)$ vanishes on $\mathcal{K}_{p}^{0 ; \mp}$, and $\mathrm{F}_{p}^{0} h(\boldsymbol{w}, q)=0$ for every $q>s>0$ and $\boldsymbol{w} \in \mathcal{S}^{n-1}$, then $h(e \boldsymbol{u})$ vanishes for every $\mathrm{e}>|-p \pm 1| q$ and $\boldsymbol{u} \in \mathcal{S}^{n-1}$.

$\langle\mathrm{sp} 3\rangle \quad$ If $h \in C_{\infty}\left(\mathcal{K}_{0}^{n}\right)$ vanishes on $\mathcal{K}_{\infty}^{0 ; \mp}$, and $\mathrm{F}_{\infty}^{0} h(\boldsymbol{w}, q)=0$ for every $q>s>0$ and $\boldsymbol{w} \in \mathcal{S}^{n-1}$, then $h(e \boldsymbol{u})$ vanishes for every $e>s$ and $\boldsymbol{u} \in \mathcal{S}^{n-1}$.

Theorem 6.2 is a direct application of Support Theorem 2.1, so we only notice that in statement $\langle$ sp1 $\rangle$ we can also deduce that $S_{ \pm}^{0} h( \pm 1 ; \boldsymbol{w}, 0)=0$.

Finally, we deal with the hyperbolic case. Observe that for $p= \pm 1$ there cannot exist support theorem in the usual sense. The reason behind this is that if, say, $p=-1$, then $\bar{\Psi}_{-1}^{-1 ;-}$ maps the lower sheet $\check{\mathcal{K}}_{-1}^{n}$ of the hyperboloid $\mathcal{K}_{-1}^{n}$ onto the complement of the unit ball in $\mathcal{A}_{0}^{n}$ in such a way that the points of "infinity" in $\check{\mathcal{K}}_{-1}^{n}$ are sent to the unit sphere in $\mathcal{A}_{0}^{n}$. In the same way, if $p=1$, then $\bar{\Psi}_{-1}^{1 ;+}$ maps the upper sheet $\hat{\mathcal{K}}_{-1}^{n}$ of the hyperboloid $\mathcal{K}^{n}$ onto the complement of the unit ball in $\mathcal{A}_{2}^{n}$ in such a way that the points of "infinity" in $\overline{\mathcal{K}}_{-1}^{h}$ are sent to the unit sphere in $\mathcal{A}_{2}^{n}$. 
Theorem 6.3 Support theorems for shifted Funk transforms on the hyperboloid $\mathcal{K}_{-1}^{n}$

$\langle\operatorname{sh} 1\rangle \quad$ If $h \in C_{\infty}\left(\mathcal{K}_{-1}^{n}, \pm 1\right)$ and $\mathrm{F}_{ \pm 1}^{-1} h(\boldsymbol{w}, q)=0$ for every $q>s>1$ and $\boldsymbol{w} \in \mathcal{S}^{n-1}$, then $h\left(\operatorname{Exp}_{O^{ \pm}}(\right.$eu $\left.)\right)$vanishes for every e $<2 \operatorname{artanh}(1 / s)$ and $\boldsymbol{u} \in \mathcal{S}^{n-1}$.

$\langle\operatorname{sh} 2\rangle \quad$ If $h \in C_{n}\left(\mathcal{K}_{-1}^{n}, 0\right)$ vanishes either on $\breve{\mathcal{K}}_{-1}^{n}$ or on $\hat{\mathcal{K}}_{-1}^{n}$, and $\mathrm{F}_{0}^{-1} h(\boldsymbol{w}, q)=0$ for every $q>s \in(0,1)$ and $\boldsymbol{w} \in \mathcal{S}^{n-1}$, then $h\left(\operatorname{Exp}_{O^{ \pm}}(e \boldsymbol{u})\right)$ vanishes for every $e>2 \operatorname{artanh}(s)$ and $\boldsymbol{u} \in \mathcal{S}^{n-1}$.

$\langle\operatorname{sh} 3\rangle \quad$ If $|p| \neq 0$, and $h \in C_{n-1}\left(\mathcal{K}_{-1}^{n}, p\right)$ vanishes on $\check{\mathcal{K}}_{-1}^{n}$ or on $\hat{\mathcal{K}}_{-1}^{n}$ according to $p<0$ or $p>0$, respectively, and $\mathrm{F}_{p}^{-1} h(\boldsymbol{w}, q)=0$ for every $q>s>0$ and $\boldsymbol{w} \in \mathcal{S}^{n-1}$, then $h\left(\operatorname{Exp}_{O^{ \pm}}(\right.$eu $\left.)\right)$vanishes for every e $>\delta_{p}^{-1 ; \pm}(s)$ and $\boldsymbol{u} \in \mathcal{S}^{n-1}$.

$\langle\operatorname{sh} 4\rangle \quad$ If $h \in C_{\infty}\left(\mathcal{K}_{-1}^{n}\right)$ vanishes either on $\check{\mathcal{K}}_{-1}^{n}$ or on $\hat{\mathcal{K}}_{-1}^{n}$ and $\mathrm{F}_{\infty}^{-1} h(\boldsymbol{w}, q)=0$, for every $q>s>0$ and $\boldsymbol{w} \in \mathcal{S}^{n-1}$, then $h\left(\operatorname{Exp}_{O^{ \pm}}(e \boldsymbol{u})\right)$ vanishes for every $e>\operatorname{arsinh}(s)$ and $\boldsymbol{u} \in \mathcal{S}^{n-1}$.

Proof We prove the statements one after the other.

$\left\langle\right.$ sh1〉 From (4.2) we have $\mathcal{M}_{-1 ; \pm 1}^{n}=\mathbb{R}^{n}$. By symmetry, it is enough to prove for $p=-1$. So, according to (6.1) and (4.15), for $q>1$ we have $\mathrm{F}_{-1}^{-1} h(\boldsymbol{w}, q)=\mathrm{S}_{-}^{-1} h(-1 ; \boldsymbol{w}, q)$. Let $f=\mathrm{N}_{-1}^{-1 ;-}\left(h \circ \Psi_{-1}^{-1 ;-}\right)$. Then $f \in C_{\infty}\left(\mathbb{R}^{n}\right)$ as $h \in C_{\infty}\left(\mathcal{K}_{-1}^{-1},-1\right)$. Further, by (5.4), $f$ satisfies $\mathrm{S}_{-}^{-1} h(-1 ; \boldsymbol{w}, q)=\mathrm{R} f(\boldsymbol{w}, q)$, hence $\mathrm{R} f$ vanishes for $q>s(s>1)$. So, by Support Theorem 2.1, $f$ vanishes for $|\boldsymbol{x}|>s>1$. Thus $h$ vanishes for $\Psi_{-1}^{-1 ;-}(\boldsymbol{x})$ if $|\boldsymbol{x}|>s$, hence the statement follows from (6.3) and (6.4).

$\langle\operatorname{sh} 2\rangle \quad$ Firstly, we observe that $\mathcal{M}_{1 ; p}^{n}=\mathcal{B}^{n}$ by (4.2). We assume that $h$ vanishes on $\check{\mathcal{K}}_{-1}^{n}$. So, according to (6.1) and (4.15), we have $\mathrm{F}_{0}^{-1} h(\boldsymbol{w}, q)=\mathrm{S}_{+}^{-1} h(0 ; \boldsymbol{w}, q)$. Let $f=\mathrm{N}_{0}^{-1 ;+}\left(h \circ \Psi_{0}^{-1 ;+}\right)$. Then $f(\boldsymbol{x})=\frac{1}{\sqrt{1-x^{2}}} h\left(\Psi_{0}^{-1 ;+}(\boldsymbol{x})\right)$, hence $f \in C_{0}\left(\mathcal{B}^{n}\right)$ because $h \in C_{n}\left(\mathcal{K}_{-1}^{n}, 0\right)$. Further, by (5.4), $f$ satisfies $\mathrm{S}_{+}^{-1} h(0 ; \boldsymbol{w}, q)=\sqrt{1-q^{2}} \mathrm{R} f(\boldsymbol{w}, q)$, hence $\operatorname{Rf}$ vanishes for $q>s \in(0,1)$. So, by Support Theorem 2.1, $f$ vanishes for $|x|>s$. Thus $h$ vanishes for $\Psi_{0}^{-1 ;+}(\boldsymbol{x})$ if $|\boldsymbol{x}|>s$, hence the statement follows from (6.3) and

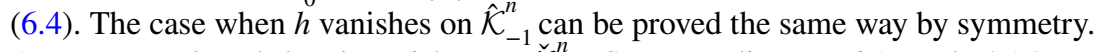

$\langle\operatorname{sh} 3\rangle$ Assume $p<0$ and that $h$ vanishes on $\check{\mathcal{K}}_{-1}^{n}$. So, according to (6.1) and (4.15), we have $\mathrm{F}_{p}^{-1} h(\boldsymbol{w}, q)=\mathrm{S}_{+}^{-1} h(p ; \boldsymbol{w}, q)$. Let $f=\mathrm{N}_{p}^{-1 ;+}\left(h \circ \Psi_{p}^{-1 ;+}\right)$. Then $f \in C_{0}\left(\mathcal{B}^{n}\right)$ because $h \in C_{n-1}\left(\mathcal{K}_{-1}^{n}, p\right)$. Further, by (5.4), $f$ satisfies $\mathrm{S}_{+}^{-1} h(p ; \boldsymbol{w}, q)=\sqrt{1-q^{2}} \mathrm{R} f(\boldsymbol{w}, q)$, hence $\mathrm{R} f$ vanishes for $q>s \in(0,1)$. So, by Support Theorem 2.1, $f$ vanishes for $|\boldsymbol{x}|>s$. Thus $h$ vanishes for $\Psi_{p}^{-1 ;+}(\boldsymbol{x})$ if $|\boldsymbol{x}|>s$, hence the statement follows from (6.3) and (6.4). The case when $p>0$ can be proved the same way by symmetry.

$\left\langle\right.$ sh4 Firstly, we observe that $\mathcal{M}_{1 ; \infty}^{n}=\mathbb{R}^{n}$ by (4.2). We assume that $h$ vanishes on $\check{\mathcal{K}}_{-1}^{n}$. So, according to (6.1) and (4.15), we have $\mathrm{F}_{\infty}^{-1} h(\boldsymbol{w}, q)=\mathrm{S}_{+}^{-1} h(\infty ; \boldsymbol{w}, q)$. Let $f=\mathrm{N}_{\infty}^{-1 ;+}\left(h \circ \Psi_{\infty}^{-1 ;+}\right)$. Then $f \in C_{\infty}\left(\mathbb{R}^{n}\right)$ because $h \in C_{\infty}\left(\mathcal{K}_{-1}^{n}, \infty\right)$. Further, by (5.5), $f$ satisfies $\mathrm{S}_{+}^{-1} h(p ; \boldsymbol{w}, q)=\sqrt{1+q^{2}} \mathrm{R} f(\boldsymbol{w}, q)$, hence $\mathrm{R} f$ vanishes for $q>s>0$. So, by Support Theorem 2.1, $f$ vanishes for $|\boldsymbol{x}|>s$. Thus $h$ vanishes for $\Psi_{\infty}^{-1 ;+}(\boldsymbol{x})$ if $|\boldsymbol{x}|>s$, hence the statement follows from (6.3) and (6.4). The case when $h$ vanishes on $\hat{\mathcal{K}}_{-1}^{n}$ can be proved the same way by symmetry.

Statement $\langle\operatorname{sh} 1\rangle$, the first result about the hyperbolic slice transform, is not valid for $s \leq 1$, but $\langle$ sh3 $\rangle$ gives appropriate support theorem on a sheet of $\mathcal{K}_{-1}^{n}$.

Statement $\langle\operatorname{sh} 2\rangle$ is just a reformulation of [24, $\left(\mathrm{i}^{-}\right)$in Theorem 3.2]. 
Important to know that the decay conditions cannot be dropped from any of these theorems as shown in counter examples (see [18, Remark 2.9 of Chapter I]).

\subsection{Kernel descriptions}

Let $h$ be a continuous function on $\mathcal{K}_{\kappa}^{n}$. Using (5.2) we define the functions

$$
h_{p}^{ \pm}: \mathcal{K}_{\kappa}^{n} \ni E \mapsto h_{p}^{ \pm}(E)= \begin{cases}0 & \text { if } E \notin \mathcal{K}_{p}^{\kappa ; \pm}, \\ h(E) & \text { if } E \in \mathcal{K}_{p}^{\kappa ; \pm},\end{cases}
$$

for $p \in(\mathbb{R} \backslash\{ \pm 1\}) \cup\{ \pm \infty\}$. If $h \in C_{k}\left(\mathcal{K}_{\kappa}^{n}, p\right)$ for $k \in \mathbb{N}$, then, obviously, both functions $h_{p}^{ \pm}$ are in $C_{k}\left(\mathcal{K}_{\kappa}^{n}, p\right)$.

We start considering the kernels in the elliptic case. This makes a direct generalization of Funk's result [13] and leads to kernel descriptions different than the ones in [7, $15,16,20,26,27,30,31,33,34]$. Figure 4 shows what is at stake.

Theorem 6.4 Kernels of some shifted Funk transform on the sphere $\mathcal{K}_{1}^{n}$.

$\langle\mathrm{ks} 1\rangle \quad$ If $h \in C_{\infty}\left(\mathcal{K}_{1}^{n}, \pm 1\right)$, then $\mathrm{F}_{+1}^{1} h$ vanishes if and only if $h \equiv 0$.

$\langle\mathrm{ks} 2\rangle \quad$ If $|p|<1$ and $h \in C_{\infty}\left(\mathcal{K}_{1}^{n}, p\right)$, then $\mathrm{F}_{p}^{1} h$ vanishes if and only if there is a function $f \in C_{\infty}\left(\mathcal{M}_{1 ; p}^{n}\right)$ such that $\pm h_{p}^{ \pm} \circ \Psi_{p}^{1 ; \pm}=\overline{\mathbf{N}}_{p}^{1 ; \pm} f$.

$\langle\mathrm{ks} 3\rangle$ If $|p|>1$ and $h \in C\left(\mathcal{K}_{1}^{n}, p\right)$, then $\mathrm{F}_{p}^{1} h$ vanishes if and only if there is a function $f \in C\left(\mathcal{M}_{1 ; p}^{n}\right)$ such that $\pm h_{p}^{ \pm} \circ \Psi_{p}^{1 ; \pm}=\overline{\mathrm{N}}_{p}^{1 ; \pm} f$.

$\langle\mathrm{ks} 4\rangle \quad$ If $h \in C\left(\mathcal{K}_{1}^{n}, \infty\right)$, then $\mathrm{F}_{\infty}^{1} h$ vanishes if and only if there is a function $f \in C\left(\mathcal{M}_{1 ; \infty}^{n}\right)$ such that $\pm h_{\infty}^{ \pm} \circ \Psi_{\infty}^{1 ; \pm}=f$.

Proof Statement $\langle\mathrm{ks} 1\rangle$ comes immediately from $\langle$ se 1$\rangle$.

$\langle$ ks2 $\rangle \quad$ As $|p|<1$, we have $\mathcal{M}_{1 ; p}^{n}=\mathbb{R}^{n}$ by (4.2). Let $f \in C_{\infty}\left(\mathcal{M}_{1 ; p}^{n}\right)$ and $h^{ \pm} \circ \Psi_{p}^{1 ; \pm}=\overline{\mathrm{N}}_{p}^{1 ; \pm} f$. Define the function

$$
h: \mathcal{K}_{1}^{n} \ni E \mapsto \begin{cases}h^{+}(E) & \text { if } E \in \mathcal{K}_{p}^{\kappa ;+}, \\ -h^{-}(E) & \text { if } E \in \mathcal{K}_{p}^{\kappa ;-}\end{cases}
$$
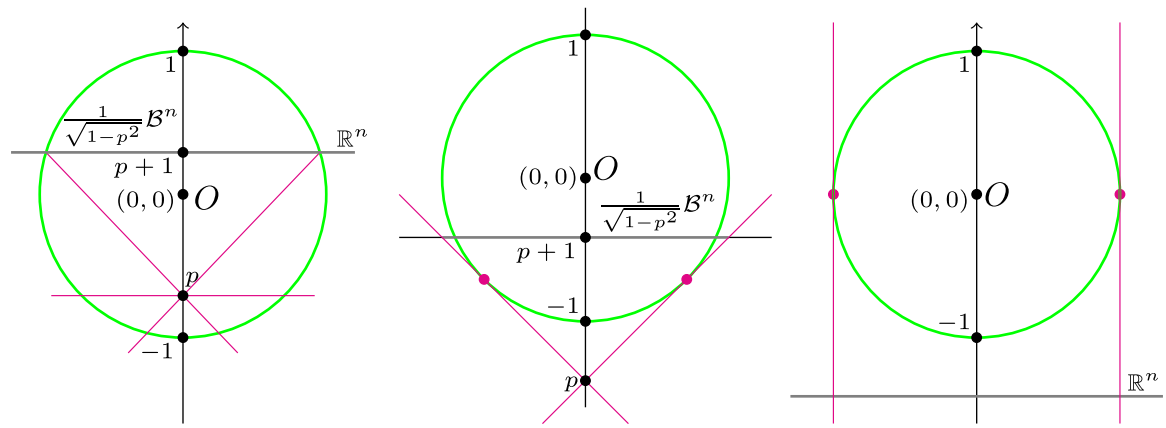

Fig. 4 Mappings $\Psi_{p}^{1 ; \pm}$ if $|p|<1,|p|>1$, and $p=\infty$ 
Then $h \in C_{\infty}\left(\mathcal{K}_{1}^{n}, p\right)$ by (5.1) and (5.3), and $\mathrm{F}_{p}^{1} h=0$ by (5.4), (6.8), (6.1) and (4.15). Further, $\pm h_{p}^{ \pm} \circ \Psi_{p}^{1 ; \pm}= \pm h^{ \pm} \circ \Psi_{p}^{1 ; \pm}=\overline{\mathrm{N}}_{p}^{1 ; \pm} f$.

If $h \stackrel{p}{\in} C_{\infty}^{p}\left(\mathcal{K}_{1}^{n}, p\right)$ and $\stackrel{p}{F}_{p}^{1} h$ vanishes, then $h_{p}^{ \pm} \in C_{\infty}\left(\mathcal{K}_{1}^{n}, p\right)$, and $\mathrm{F}_{p}^{1} h_{p}^{-}=-\mathrm{F}_{p}^{1} h_{p}^{+}$because $0=\mathrm{F}_{p}^{1} h=\mathrm{F}_{p}^{1} h_{p}^{+}+\mathrm{F}_{p}^{1} h_{p}^{-}$. So, by Theorem 5.1, we have $0=\mathrm{R} \ell_{+}+R^{p} \ell_{-}$for the functions $\ell_{ \pm}=\mathrm{N}_{p}^{1 ; \pm}\left(h_{p}^{ \pm} \circ \Psi_{p}^{1 ; \pm}\right)$. As the functions $\ell_{ \pm}$are in $C\left(\mathbb{R}^{n}\right)$, Support Theorem 2.1 implies $\ell_{-}=-\ell_{+}$. Then for $f=\ell_{+}$we have $\pm f^{ \pm}=h_{p}^{ \pm} \circ \Psi_{p}^{1 ; \pm}$, hence the statement.

$\langle\mathrm{ks} 3\rangle$ and $\langle\mathrm{ks} 4\rangle$ : By (4.2) we have $\mathcal{M}_{1 ; p}^{n}=\frac{1}{\sqrt{p^{2}-1}} \mathcal{B}^{n}$ for $|p|>1$, and $\mathcal{M}_{1 ; \infty}^{n}=\mathcal{B}^{n}$. Since these are compact sets, the reasoning given for $\langle$ ks 2$\rangle$ works very well for these statements too, without the condition of infinite decay, so we leave the details to the interested reader.

Notice that $\langle\mathrm{ks} 1\rangle$ states the injectivity of the spherical slice transform under a mild condition, while the next statements describes the kernels of the spherical shifted Funk transform by a kind of parity condition in accordance with the results of $[3,4,6,7$, $20,26,30,33,34,38]$. Since $\langle\mathrm{ks} 2\rangle$ served as a prototype for the next statements of the theorem, it is certainly not the sharpest possible version, so we return to it in Sect. 9, where the sharp version $\left\langle\mathrm{ks} 2{ }^{\prime}\right\rangle$ is proved by applying our intertwining relations (5.4) and Funk's theorem about the Funk transform [13].

Although the result does not add very much new to the theory, we continue with the parabolic case for the sake of completeness.

Theorem 6.5 Kernels of some shifted Funk transforms on $\mathcal{K}_{0}^{n}$ :

$\langle\mathrm{kp} 1\rangle \quad$ If $h \in C_{\infty}\left(\mathcal{K}_{0}^{n}\right)$, then $\mathrm{F}_{ \pm 1}^{0} h$ vanishes if and only if $h$ vanishes on $\mathcal{K}_{ \pm 1}^{0 ; \mp}$, and the integrals of $h$ over hyperplanes through $O^{ \pm}$in $\mathcal{K}_{\mp 1}^{0 ; \mp}$ vanish.

$\langle\mathrm{kp} 2\rangle \quad$ If $|p| \neq 1$ and $h \in C_{\infty}\left(\mathcal{K}_{0}^{n}\right)$, then $\mathrm{F}_{p}^{0} h$ vanishes if and only if there is a function $f \in C_{\infty}\left(\mathcal{M}_{0 ; p}^{n}\right)$ such that $\pm h_{p}^{ \pm} \circ \Psi_{p}^{0 ; \pm}{ }^{p}=\overline{\mathbf{N}}_{p}^{0 ; \pm} f$.

$\langle\mathrm{kp} 3\rangle \quad$ If $h \in C_{\infty}\left(\mathcal{K}_{0}^{n}\right)$, then $\mathrm{F}_{\infty}^{0} h$ vanishes if and only if there is a function $f \in C\left(\mathcal{M}_{0 ; \infty}^{n}\right)$ such that $\pm h_{\infty}^{ \pm} \circ \Psi_{\infty}^{0 ; \pm}=f$.

Proof $\langle\mathrm{kp} 1\rangle$ : It is clear that $\mathrm{F}_{p}^{0} h$ vanishes if $h$ vanishes on $\mathcal{K}_{p}^{0 ; \mp}$, and the integrals of $h$ over hyperplanes through $O^{ \pm}$in $\mathcal{K}_{-p}^{0 ; \mp}$ vanish.

For the "only if" part of the statement we only need to prove for $p=-1$ by the symmetry.

Let $\mathcal{H}_{\boldsymbol{u}}$ be the 1-codimensional subspace of $\mathcal{A}_{-1}^{n}$ orthogonal to $\boldsymbol{u} \in \mathcal{S}^{n} \cap \mathcal{A}_{-1}^{n}$. Let $g(\boldsymbol{u})$ be the integral of $h$ over $\mathcal{H}_{\boldsymbol{u}}$. Then $\mathrm{R} h^{+}(\boldsymbol{u}, r)+g(\boldsymbol{u}) \equiv 0$ by (2.2). However, by (2.2), $\mathrm{R} h^{+}(\boldsymbol{u}, r) \rightarrow 0$ if $r \rightarrow \infty$, so we deduce $g(\boldsymbol{u}) \equiv 0$. Then $\mathrm{R} h^{+}(\boldsymbol{u}, r) \equiv 0$ follows from which Support Theorem 2.1 implies the statement.

We do not give the proof of $\langle\mathrm{kp} 2\rangle$ and $\langle\mathrm{kp} 3\rangle$ here, because the procedures are very much analogous to the proof given for the elliptic case.

We finish this section with the hyperbolic case. There seems to be no previous results in the literature about the shifted Funk transform for the hyperbolic case. However, for the hyperbolic Funk transform and for the hyperbolic slice transforms, the results seem greatly analogous to the spherical case. Figure 5 shows what the next theorem is about. 

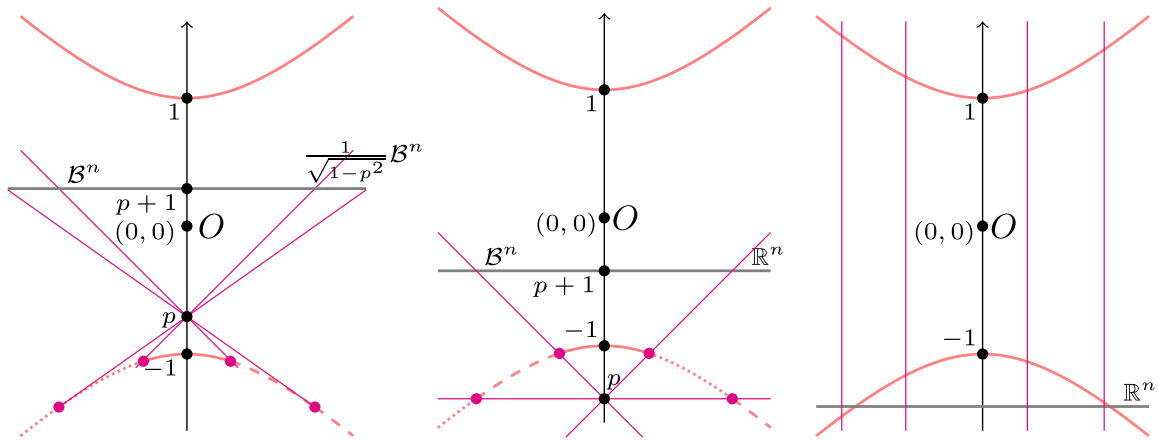

Fig. 5 Mappings $\Psi_{p}^{-1 ; \pm}$ if $|p|<1,|p|>1$, and $p=\infty$

Theorem 6.6 Kernels of some shifted Funk transform on the hyperboloid $\mathcal{K}_{-1}^{n}$.

$\langle\mathrm{kh} 1\rangle \quad$ If $h \in C_{\infty}\left(\mathcal{K}_{-1}^{n}, \pm 1\right)$, then $\mathrm{F}_{ \pm 1}^{-1} h$ vanishes if and only if $h \equiv 0$.

$\langle\mathrm{kh} 2\rangle \quad$ If $h \in C_{n}\left(\mathcal{K}_{-1}^{n}\right)$, then $\mathrm{F}_{0}^{-1} h$ vanishes if and only if there is a function $f \in C\left(\mathcal{M}_{-1 ; 0}^{n}\right)$ such that $\pm h_{0}^{ \pm} \circ \Psi_{0}^{-1 ; \pm}=\overline{\mathrm{N}}_{0}^{-1 ; \pm} f$.

$\langle\mathrm{kh} 3\rangle$ If $|p| \in(0,1)$ and $h \in C_{n-1}\left(\mathcal{K}_{-1}^{n}, p\right)$, then $\mathrm{F}_{p}^{-1} h$ vanishes if and only if there is a function $f \in C_{0}\left(\mathcal{M}_{-1 ; p}^{n}\right) \cap C_{0}\left(\mathcal{M}_{-1 ; 0}^{n}\right)$ such that $\pm h_{p}^{ \pm} \circ \Psi_{p}^{-1 ; \pm}=\overline{\mathrm{N}}_{p}^{-1 ; \pm} f$.

$\langle\mathrm{kh} 4\rangle \quad$ If $|p|>1$ and $h \in C_{\infty}\left(\mathcal{K}_{-1}^{n}, p\right)$, then $\mathrm{F}_{p}^{-1} h$ vanishes if and only if there is a function $f \in C\left(\mathcal{M}_{-1 ; p}^{n}\right)$ such that $\pm h_{p}^{ \pm} \circ \Psi_{p}^{-1 ; \pm}=\overline{\mathbf{N}}_{p}^{-1 ; \pm} f$.

$\langle\mathrm{kh} 5\rangle \quad$ If $h \in C_{\infty}\left(\mathcal{K}_{-1}^{n}\right)$, then $\mathrm{F}_{\infty}^{-1} h$ vanishes if and only if there is a function $f \in C\left(\mathcal{M}_{-1 ; \infty}^{n}\right)$ such that $\pm h_{\infty}^{ \pm} \circ \Psi_{\infty}^{-1 ; \pm}=f$.

Proof We prove the statements one after the other.

$\langle\mathrm{kh} 1\rangle$ From $\langle\operatorname{sh} 1\rangle$ we get that $h\left(\operatorname{Exp}_{O^{ \pm}}(e \boldsymbol{u})\right)$ vanishes for every $e<2 \operatorname{artanh}(1)=\infty$ and $\boldsymbol{u} \in \mathcal{S}^{n-1}$, i.e., $h$ vanishes on $\hat{\mathcal{K}}_{-1}^{n}$. From $\langle$ sh3 $\rangle$ we obtain that $h$ vanishes on $\check{\mathcal{K}}_{-1}^{n}$ if $\mathrm{F}_{1}^{-1}$ is under consideration, or on $\hat{\mathcal{K}}_{-1}^{n}$ if $\mathrm{F}_{-1}^{-1}$ is under consideration.

$\langle\operatorname{kh} 2\rangle \quad$ We have $\mathcal{M}_{-1 ; 0}^{n}=\mathcal{B}^{n}$ by (4.2). Let $f \in C_{0}\left(\mathcal{M}_{-1 ; 0}^{n}\right)$ and define the function

$$
h: \mathcal{K}_{-1}^{n} \ni E \mapsto \begin{cases}h^{+}(E) & \text { if } E \in \Psi_{0}^{-1 ;+}\left(\mathcal{M}_{-1 ; 0}^{n}\right) \\ -h^{-}(E) & \text { if } E \in \Psi_{0}^{-1 ;-}\left(\mathcal{M}_{-1 ; 0}^{n}\right)\end{cases}
$$

where $h^{ \pm} \circ \Psi_{0}^{-1 ; \pm}=\overline{\mathrm{N}}_{0}^{-1 ; \pm} f$, i.e., $h^{ \pm}\left(\Psi_{0}^{-1 ; \pm}(\boldsymbol{x})\right)=f(\boldsymbol{x}){\sqrt{1-\boldsymbol{x}^{2}}}^{n}$. Then $h \in C_{n}\left(\mathcal{K}_{-1}^{n}, 0\right)$, and $\mathrm{F}_{0}^{-1} h=0$ by (6.1), (4.15), and Theorem 5.1. Further, $\pm h_{0}^{ \pm} \circ \Psi_{0}^{-1 ; \pm}=h^{ \pm} \circ \Psi_{0}^{-1 ; \pm}=\overline{\mathrm{N}}_{0}^{-1 ; \pm} f$.

If $h \in C_{n}\left(\mathcal{K}_{-1}^{n}, 0\right)$ and $\mathrm{F}_{0}^{-1} h$ vanishes, then $h_{0}^{ \pm} \in C_{n}\left(\mathcal{K}_{-1}^{n}, 0\right)$, and $\mathrm{F}_{0}^{-1} h_{0}^{-}=-\mathrm{F}_{0}^{-1} h_{0}^{+}$ because $0=\mathrm{F}_{0}^{-1} h=\mathrm{F}_{0}^{-1} h_{0}^{+}+\mathrm{F}_{0}^{-1} h_{0}^{-}$. So, by Theorem 5.1, we have $0=R g_{+}+R g_{-}$for the functions $g_{ \pm}=\mathrm{N}_{0}^{-1 ; \pm} h_{0}^{ \pm} \circ \Psi_{0}^{-1 ; \pm}$ which are in $C\left(\mathcal{B}^{n}\right)$, because $g_{ \pm}(x)=\frac{1}{\sqrt{1-x^{2}}} h_{0}^{ \pm}\left(\Psi_{0}^{-1 ; \pm}(\boldsymbol{x})\right)$. So Support Theorem 2.1 implies $g_{-}=-g_{+}$. Let $f=g_{+}$. Then we get $\pm \overline{\mathrm{N}}_{0}^{-; \pm} f=h_{0}^{ \pm} \circ \Psi_{0}^{-1 ; \pm}$, hence the statement.

$\langle\mathrm{kh} 3\rangle$ : Equation (4.2) gives $\mathcal{M}_{-1 ; p}^{n}=\frac{1}{\sqrt{1-p^{2}}} \mathcal{B}^{n}$ for $|p| \in(0,1)$. Since this is a compact set, the reasoning given for $\langle\mathrm{kh} 2\rangle$ works very well for this statements too, but needs only a decay of order $n-1$, so we leave the details to the reader. 
$\langle\mathrm{kh} 4\rangle$ and $\langle\mathrm{kh} 5\rangle$ : Equation (4.2) gives $\mathcal{M}_{-1 ; p}^{n}=\mathbb{R}^{n}$ for $|p|>1$ and $\mathcal{M}_{-1 ; \infty}^{n}=\mathbb{R}^{n}$. It is clear that the reasoning given for $\langle\mathrm{kh} 2\rangle$ works very well in these cases too, but with infinite decay condition, so we leave the details to the interested reader.

According to $\langle\mathrm{kh} 1\rangle$ the hyperbolic slice transform is injective on $C_{\infty}\left(\mathcal{K}_{-1}^{n}, \pm 1\right)$, while the kernel of the hyperbolic Funk transform is the set of odd functions in $C_{n}\left(\mathcal{K}_{-1}^{n}\right)$ by $\langle\operatorname{kh} 2\rangle$. These results are totally analogous to the case of the sphere.

\section{Funk-type isodistant Radon transforms}

The double covering of $\mathbb{K}_{K}^{n}$ given by (1.3) can be reduced by considering the identifying mapping $\hat{\chi}_{\kappa}: \hat{\mathcal{K}}_{\kappa}^{n} \ni E \rightarrow(E,-E) \in \overline{\mathcal{K}}_{\kappa}^{n} \cong \mathbb{K}_{\kappa}^{n}$. Then $\hat{\chi}_{\kappa}$ is bijective for $\kappa \leq 0$ as well as for $\kappa=1$ if the totally geodesic corresponding to $\mathcal{K}_{1}^{n} \cap \mathcal{A}_{0}^{n}$ is left out. If $h \in C\left(\mathbb{K}_{\kappa}^{n}\right)$, then the corresponding function on $\mathcal{K}_{\kappa}^{n}$ is

$$
\hat{h}: \mathcal{K}_{\kappa}^{n} \ni E \rightarrow \hat{h}(E)= \begin{cases}h\left(\hat{\chi}_{\kappa}(E)\right) & \text { if } E \in \hat{\mathcal{K}}_{\kappa}^{n}, \\ 0 & \text { otherwise. }\end{cases}
$$

We define the Funk-type isodistant Radon transform $\hat{\mathrm{R}}_{p}^{\kappa}$ of a suitable function $h \in C\left(\mathbb{K}_{\kappa}^{n}\right)$ by

$$
\begin{array}{cc}
\hat{\mathrm{R}}_{p}^{\kappa} h(\boldsymbol{w}, g)=\mathrm{F}_{p}^{\kappa} \hat{h}\left(\boldsymbol{w}, \tau_{\kappa}(g)\right) & \text { if } p \in \mathbb{R}, \\
\hat{\mathrm{R}}_{\infty}^{\kappa} h(\boldsymbol{w}, \varrho)=\mathrm{F}_{\infty}^{\kappa} \hat{h}\left(\boldsymbol{w}, \sigma_{\kappa}(\varrho)\right) & \text { if } p=\infty,
\end{array}
$$

where $\boldsymbol{w} \in \mathcal{S}^{n-1}, g, \varrho \in\left[0, \rho_{\kappa}\right)$. So the Funk-type isodistant Radon transform $\hat{\mathrm{R}}_{p}^{\kappa}$ is essentially the restriction of the shifted Funk transform to the set of hyperplanes intersecting $\hat{\mathcal{K}}_{\kappa}^{n}$ in isodistants.

For our considerations we will need decay conditions, so we introduce the function space $C_{m}\left(\mathbb{K}_{\kappa}^{n}, \hat{\chi}_{\kappa}(\mathcal{L})\right)$ so that $h \in C_{m}\left(\mathbb{K}_{\kappa}^{n}, \hat{\chi}_{\kappa}(\mathcal{L})\right)$ if $h \circ \hat{\chi}_{\kappa} \in C_{m}\left(\hat{\mathcal{K}}_{\kappa}^{n}, \mathcal{L}\right)(m \in \mathbb{N})$, where $\mathcal{L} \subset \hat{\mathcal{K}}_{\kappa}^{n}$ is a nonempty, open domain. Further, we define $C_{m}\left(\mathbb{K}_{\kappa}^{n}\right)$ so that $h \in C_{m}\left(\mathbb{K}_{\kappa}^{n}\right)$ if $h \circ \hat{\chi}_{\kappa} \in C_{m}\left(\hat{\mathcal{K}}_{\kappa}^{n}\right)(m \in \mathbb{N})$. Additionally, analogously to the notations after (6.6), we use the notation $C_{m}\left(\mathbb{K}_{\kappa}^{n}, p\right):=C_{m}\left(\mathbb{K}_{\kappa}^{n}, \hat{\chi}_{\kappa}\left(\mathcal{K}_{p}^{\kappa ; \pm}\right)\right)$ too (see (5.2)). We also need to define the functions

$$
\hat{h}_{p}^{ \pm}: \hat{\mathcal{K}}_{\kappa}^{n} \ni E \mapsto \hat{h}_{p}^{ \pm}(E)= \begin{cases}0 & \text { if } E \notin \Psi_{p}^{\kappa ; \pm}\left(\mathcal{M}_{\kappa ; p}^{n}\right), \\ h\left(\hat{\chi}_{\kappa}(E)\right) & \text { if } E \in \Psi_{p}^{\kappa ; \pm}\left(\mathcal{M}_{\kappa ; p}^{n}\right)\end{cases}
$$

for every $h \in \mathbb{K}_{\kappa}^{n}$, where $p \in \mathbb{R} \cup\{ \pm \infty\}$, and $\Psi_{p}^{\kappa ; \pm}$ is given by (5.1). Observe that if $h \in C_{k}\left(\mathbb{K}_{\kappa}^{n}, p\right)$, then both functions $\hat{h}_{p}^{ \pm}$are in $C_{k}\left(\hat{\mathcal{K}}_{\kappa}^{n}, p\right)$ for every $k \in \mathbb{N}$.

In the elliptic case, every slice of $\hat{\mathcal{K}}_{1}^{n}$ is a part of an isodistant in $\mathcal{K}_{1}^{n}$, so the properties of $\hat{\mathrm{R}}_{p}^{1}$ are essentially similar to that of $\mathrm{F}_{p}^{1}$. We give these properties without proof, because they follow directly from theorems 6.1 and 6.4 with the use of the functions $\delta_{p}^{\kappa ; \pm}$ defined in (6.2). 
Theorem 7.1 The Funk-type isodistant Radon transform in the elliptic space have the following properties:

$\left\langle\right.$ ee1 $\quad$ If $p<0, d \in[0, \arctan (1 / p)), h \in C\left(\mathbb{K}_{1}^{n}\right)$ and $\hat{\mathrm{R}}_{p}^{1} h(\boldsymbol{w}, g)=0$ for every $g>d$ and $\boldsymbol{w} \in \mathcal{S}^{n-1}$, then $h\left(\operatorname{Exp}_{O^{+}}(\right.$eu $\left.)\right)$vanishes for every $e^{p} \delta_{p}^{1 ;+}(\tan (d))$ and $\boldsymbol{u} \in \mathcal{S}^{n-1}$.

$\langle\mathrm{ee} 2\rangle \quad$ If $d \in[0, \pi / 2), h \in C_{\infty}\left(\mathbb{K}_{1}^{n}, 0\right)$ and $\hat{\mathrm{R}}_{0}^{1} h(\boldsymbol{w}, g)=0$ for every $g>d$ and $\boldsymbol{w} \in \mathcal{S}^{n-1}$, then $h\left(\operatorname{Exp}_{O^{+}}(\right.$eu $\left.)\right)$vanishes for every $e>d$ and $\boldsymbol{u} \in \mathcal{S}^{n-1}$.

$\left\langle\right.$ ee3 Let $p \in(0,1)$, and $h \in C_{\infty}\left(\mathbb{K}_{1}^{n}, p\right)$ is such that $h\left(\operatorname{Exp}_{O^{+}}(\right.$eu $\left.)\right)$vanishes for every $e>\arccos p$ and $\boldsymbol{u} \in \mathcal{S}^{n-1}$. If $d \in[0, \pi / 2)$, and $\hat{\mathrm{R}}_{p}^{1} h(\boldsymbol{w}, g)=0$ for every $g>d$ and $\boldsymbol{w} \in \mathcal{S}^{n-1}$, then $h\left(\operatorname{Exp}_{O^{+}}(\right.$eu $\left.)\right)=0$ for every e $>\delta_{p}^{1 ; p}(\tan (d))$ and $\boldsymbol{u} \in \mathcal{S}^{n-1}$.

〈ee4〉 If $p \in(0,1), d \in[\arccos (1 / p), \pi / 2)$, and $h \in C_{\infty}\left(\mathbb{K}_{1}^{n}, p\right)$, then $\hat{\mathrm{R}}_{p}^{1} h(\boldsymbol{w}, g)=0$ for every $g>d$ and $\boldsymbol{w} \in \mathcal{S}^{n-1}$ if and only if there is a function $f \in C_{\infty}\left(\mathcal{M}_{1 ; p}^{n}\right)$ such that $\pm \hat{h}_{p}^{ \pm} \circ \Psi_{p}^{1 ; \pm}=\overline{\mathrm{N}}_{p}^{1 ; \pm}$ f outside of $\frac{1}{p} \mathcal{B}^{n}$.

$\left\langle\right.$ ee5 $\quad$ If $d \in[0, \pi / 2), h \in C_{\infty}\left(\mathbb{K}_{1}^{n}, 1\right)$ and $\hat{\mathrm{R}}_{1}^{1} h(\boldsymbol{w}, g)=0$ for every $g>d$ and $\boldsymbol{w} \in \mathcal{S}^{n-1}$, then $h\left(\operatorname{Exp}_{O^{+}}(\right.$eu $\left.)\right)$vanishes for every e $<\delta_{1}^{1 ;-}(\tan (d))=\pi-2 d$ and $\boldsymbol{u} \in \mathcal{S}^{n-1}$.

〈ee6) Let $p>1$ and $h \in C\left(\mathbb{K}_{1}^{n}\right)$ is such that $h\left(\operatorname{Exp}_{O^{+}}(\right.$eu $\left.)\right)$vanishes for every $e>\arccos (1 / p)$ and $\boldsymbol{u} \in \mathcal{S}^{n-1}$. If $d \in[0, \arccos (1 / p))$, and $\hat{\mathrm{R}}_{p}^{1} h(\boldsymbol{w}, g)=0$ for every $g>d$ and $\boldsymbol{w} \in \mathcal{S}^{n-1}$, then $h\left(\operatorname{Exp}_{O^{+}}(e \boldsymbol{u})\right)=0$ for every $e>\delta_{p}^{1 ;+}(\tan (d))$ and $\boldsymbol{u} \in \mathcal{S}^{n-1}$.

$\left\langle\right.$ ee7 $\quad$ Ifp $>1, d \in\left[\delta_{p}^{1 ;+}(1 / p)\right.$, arccos $\left.(1 / p)\right)$, and $h \in C\left(\mathbb{K}_{1}^{n}\right)$, then $\hat{\mathrm{R}}_{p}^{1} h(\boldsymbol{w}, g)=0$ for every $g>d$ and $\boldsymbol{w} \in \mathcal{S}^{n-1}$ if and only if there is a function $f \in C\left(\mathcal{M}_{1 ; p}^{n}\right)$ such that $\pm \hat{h}_{p}^{ \pm} \circ \Psi_{p}^{1 ; \pm}=\overline{\mathrm{N}}_{p}^{1 ; \pm} f$ outside of $\frac{1}{p} \mathcal{B}^{n}$.

$\left\langle\right.$ ee8 $\quad$ If $d \in[0, \pi / 2), h \in C\left(\mathbb{K}_{1}^{n}\right)$ and $\hat{\mathrm{R}}_{\infty}^{1} h(\boldsymbol{w}, \varrho)=0$ for every $\varrho>d$ and $\boldsymbol{w} \in \mathcal{S}^{n-1}$, then $h\left(\operatorname{Exp}_{O^{+}}(\right.$eu $\left.)\right)$vanishes for every $e>d$ and $\boldsymbol{u} \in \mathcal{S}^{n-1}$.

In the parabolic case, every slice of $\hat{\mathcal{K}}_{0}^{n}$ is an isodistant, so the properties of $\hat{\mathrm{R}}_{p}^{0}$ are exactly the same as the properties of $\mathrm{F}_{p}^{0}$ (i.e., essentially a reparameterization the classical Euclidean Radon transform). These properties are given in theorems 6.2 and 6.5.

The hyperbolic case differs significantly. If a normal vector $\boldsymbol{n}$ of a hyperplane $\mathcal{P}$ fulfills $\left\langle\boldsymbol{n}, \boldsymbol{b}_{n+1}\right\rangle>1 / \sqrt{2}$, then the intersection $\mathcal{P} \cap \mathcal{K}_{-1}^{n}$ is not an isodistant, because there does not exist a totally geodesic of codimension 1 whose hyperplane's normal vector is parallel
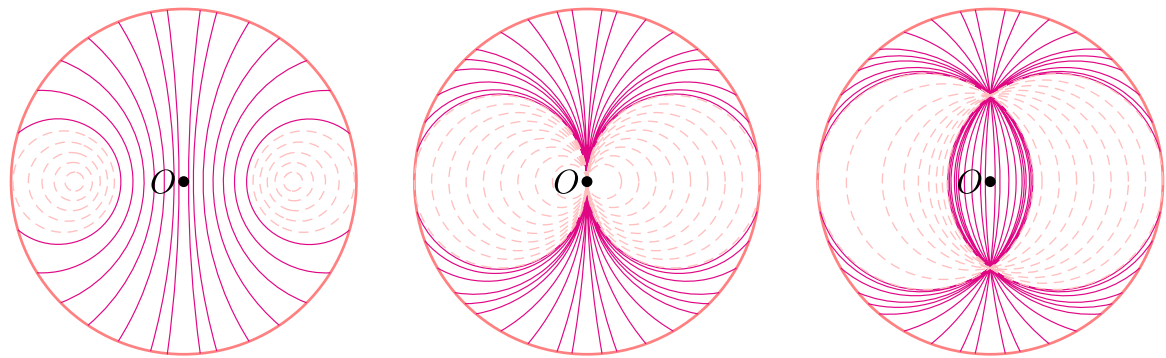

Fig. 6 Virtual and real isodistants (dashed circles vs. continuous arcs) in the Poincare disk model of the hyperbolic plane for $p \in(0,1), p=1, p>1$ 
with $\boldsymbol{n}$. These slices of $\hat{\mathcal{K}}_{-1}^{n}$ and the corresponding submanifolds in $\mathbb{K}_{-1}^{n}$, that are not isodistant, are called virtual isodistants (Fig. 6). ${ }^{4}$

Thus the properties of $\hat{\mathrm{R}}_{p}^{-1}$ have significant differences from that of $\mathrm{F}_{p}^{-1}$ while they easily follow from the statements of theorems 6.3 and 6.6.

Theorem 7.2 The Funk-type isodistant Radon transforms in the hyperbolic space have the following properties:

$\left\langle\right.$ eh1 $\quad$ If $p<0, d \in[0, \infty), h \in C_{n-1}\left(\mathbb{K}_{-1}^{n}, p\right)$, and $\hat{\mathrm{R}}_{p}^{-1} h(\boldsymbol{w}, g)=0$ for every $g>d$ and $\boldsymbol{w} \in \mathcal{S}^{n-1}$, then $h\left(\operatorname{Exp}_{O^{+}}(\right.$eu $\left.)\right)$vanishes for every e $>\delta_{p}^{-1 ;+}(\tanh (d))$ and $\boldsymbol{u} \in \mathcal{S}^{n-1}$.

$\langle\mathrm{eh} 2\rangle \quad$ If $d \in[0, \infty), h \in C_{n}\left(\mathbb{K}_{-1}^{n}, 0\right)$, and $\hat{\mathrm{R}}_{0}^{-1} h(\boldsymbol{w}, g)=0$ for every $g>d$ and $\boldsymbol{w} \in \mathcal{S}^{n-1}$, then $h\left(\operatorname{Exp}_{O^{+}}(\right.$eu $\left.)\right)$vanishes for every $e>d$ and $\boldsymbol{u} \in \mathcal{S}^{n-1}$.

〈eh3〉 Let $p \in(0,1)$ and $h \in C\left(\mathbb{K}_{-1}^{n}, p\right)$ is such that $h\left(\operatorname{Exp}_{O^{+}}(e \boldsymbol{u})\right)$ vanishes for every $e>\ln (1 / p)$ and $\boldsymbol{u} \in \mathcal{S}^{n-1}$. If $d \in[0, \ln (1 / p))$, and $\hat{\mathrm{R}}_{p}^{-1} h(\boldsymbol{w}, g)$ vanishes for every $g>d$ and $\boldsymbol{w} \in \mathcal{S}^{n-1}$, then $h\left(\operatorname{Exp}_{O^{+}}(\right.$eu $\left.)\right)=0$ for every e $>\delta_{p}^{-1 ;+}(\tanh (d))$ and $\boldsymbol{u} \in \mathcal{S}^{n-1}$.

$\langle\mathrm{eh} 4\rangle$ Let $p>1$ and $h \in C\left(\mathbb{K}_{-1}^{n}, p\right)$ is such that $h\left(\operatorname{Exp}_{O^{+}}(\right.$eu $\left.)\right)$vanishes for every $e>\ln p$ and $\boldsymbol{u} \in \mathcal{S}^{n-1}$. If $d \in[0, \ln p)$, and $\hat{\mathrm{R}}_{p}^{-1} h(\boldsymbol{w}, g)$ vanishes for every $g>d$ and $\boldsymbol{w} \in \mathcal{S}^{n-1}$, then $h\left(\operatorname{Exp}_{O^{+}}(\right.$eu $\left.)\right)=0$ for every $e^{p} \delta_{p}^{-1 ;+}(\tanh (d))$ and $\boldsymbol{u} \in \mathcal{S}^{n-1}$.

$\left\langle\right.$ eh5 $\quad$ If $d \in[0, \infty), h \in C_{\infty}\left(\mathbb{K}_{-1}^{n}\right)$, and $\hat{\mathrm{R}}_{\infty}^{-1} h(\boldsymbol{w}, \varrho)=0$ for every $\varrho>d$ and $\boldsymbol{w} \in \mathcal{S}^{n-1}$, then $h\left(\operatorname{Exp}_{O^{+}}(\right.$eu $\left.)\right)$vanishes for every $e^{>} d$ and $\boldsymbol{u} \in \mathcal{S}^{n-1}$.

Notice that no statement in this theorem is analogous to statements $\langle$ ee 4$\rangle,\langle$ ee 5$\rangle$, and $\langle$ ee7 $\rangle$ of Theorem 7.1. This is due to the fact that no virtual isodistants exist on the elliptic space.

\section{Duplex Funk-type isodistant Radon transforms}

Instead of reducing the double covering of $\mathbb{K}_{K}^{n}$ so as we did in the previous section, we can restrict the function space to the space of even functions on $\mathcal{K}_{\kappa}^{n}$. Then the isodistants of $\mathbb{K}_{\kappa}^{n}$ correspond to some of the slices of $\mathcal{K}_{\kappa}^{n}$.

We define the duplex Funk-type isodistant Radon transforms $\mathrm{R}_{p}^{\kappa}$ for suitable functions $h \in C\left(\mathbb{K}_{\kappa}^{n}\right)$ by

$$
\begin{array}{cc}
\mathrm{R}_{p}^{\kappa} h(\boldsymbol{w}, g)=\mathrm{F}_{p}^{\kappa} \tilde{h}\left(\boldsymbol{w}, \tau_{\kappa}(g)\right) & \text { if } p \in \mathbb{R}, \\
\mathrm{R}_{\infty}^{\kappa} h(\boldsymbol{w}, \varrho)=\mathrm{F}_{\infty}^{\kappa} \tilde{h}\left(\boldsymbol{w}, \sigma_{\kappa}(\varrho)\right) & \text { if } p=\infty,
\end{array}
$$

where $\boldsymbol{w} \in \mathcal{S}^{n-1}, g, \varrho \in\left[0, \rho_{\kappa}\right)$, and $\tilde{h}: \mathcal{K}_{\kappa}^{n} \ni E \mapsto h\left(\chi_{\kappa}(E)\right)$ with $\chi_{\kappa}$ given in (1.3). Recall our formula (3.3). It shows that $p$ and $g$ determine the isodistant to integrate on, more exactly $q=\tau_{\kappa}(g)$. The same formula shows that $q=\sigma_{\kappa}(\varrho)$ if $p=\infty$ (or, which is the same, $g=0)$.

Starting from any point $\boldsymbol{y}_{1}=\Psi_{p}^{\kappa ;+}\left(\boldsymbol{x}_{0}\right) \in \hat{\mathcal{K}}_{\kappa}^{n}(p \in \mathbb{R} \cup\{ \pm \infty\})$, the recursion

$$
\boldsymbol{y}_{2 i+2}:=\Psi_{p}^{\kappa ;-}\left(\boldsymbol{x}_{i}\right), \boldsymbol{y}_{2 i+3}:=-\boldsymbol{y}_{2 i+2}, \boldsymbol{x}_{i+1}:=\bar{\Psi}_{p}^{\kappa ;+}\left(\boldsymbol{y}_{2 i+3}\right) \in \mathcal{M}_{\kappa ; p}^{n}
$$

$\overline{4}$ The virtual isodistants are the horocycles and the circles. 
generates points for every $i=1,2, \ldots$. This sequence of points $\boldsymbol{y}_{i}$ is finite if $|p|=1$ or $p= \pm \infty$. Otherwise we get an infinite sequence, and it is easy to see that the sequences $\boldsymbol{y}_{2 i+1}$ and $\boldsymbol{y}_{2 i}$ tend to the points $O^{ \pm}$, respectively. (Figure 7 depicts the first points if $\kappa=1$.)

Theorem 8.1 The duplex Funk-type isodistant Radon transforms in the elliptic space have the following properties:

〈ie1〉 If $h \in C\left(\mathbb{K}_{1}^{n}\right)$, then $\mathrm{R}_{\infty}^{1} h$ vanishes if and only if there is an odd function $f \in C\left(\mathcal{M}_{1 ; \infty}^{n}\right)$ such that $\pm \tilde{h}_{\infty}^{ \pm} \circ \Psi_{\infty}^{1 ; \pm}=\overline{\mathrm{N}}_{\infty}^{1 ; \pm} f$, where $\tilde{h}=h \circ \chi_{\kappa}$.

〈ie2) If $|p|=1$, then $\mathrm{R}_{p}^{1}$ is injective on $C_{\infty}\left(\mathbb{K}_{1}^{n}, p\right)$.

〈ie3〉 If $|p|>1$, then $\mathrm{R}_{p}^{1}$ is injective on $C\left(\mathbb{K}_{1}^{n}, p\right)$.

$\left\langle\right.$ ie4〉 If $|p|<1$, then $\mathrm{R}_{p}^{1}$ is injective on $C_{\infty}\left(\mathbb{K}_{1}^{n}, p\right)$.

Proof 〈ie1〉 Let $f \in C\left(\mathcal{M}_{1 ; \infty}^{n}\right)$ be an odd function. Construct the functions $\tilde{h}^{ \pm}= \pm \overline{\mathrm{N}}_{\infty}^{1 ; \pm} f \circ \bar{\Psi}_{\infty}^{1 ; \pm}$, and then define $\tilde{h}$ by (6.8). (See the first diagram on Fig. 7.) Then $\tilde{h}$ is even, and $\mathrm{F}_{\infty}^{1} \tilde{h}$ vanishes by $\langle\mathrm{ks} 4\rangle$ of Theorem 6.4.

For the reverse direction, if $\tilde{h}$ is an even function and $\mathrm{F}_{\infty}^{1} \tilde{h}$ vanishes, then $\langle\mathrm{ks} 4\rangle$ of Theorem 6.4 implies that $\tilde{h}_{\infty}^{ \pm}= \pm \overline{\mathrm{N}}_{\infty}^{1 ; \pm} f \circ \bar{\Psi}_{\infty}^{1 ; \pm}$, hence

$$
\begin{aligned}
\overline{\mathrm{N}}_{\infty}^{1 ; \pm} f(\boldsymbol{x})=\tilde{h}_{\infty}^{+}\left(\Psi_{\infty}^{1 ;+}(\boldsymbol{x})\right) & =\tilde{h}_{\infty}^{-}\left(-\Psi_{\infty}^{1 ;+}(\boldsymbol{x})\right) \\
& =-\overline{\mathrm{N}}_{\infty}^{1 ; \pm} f\left(\bar{\Psi}_{\infty}^{1 ;-}\left(-\Psi_{\infty}^{1 ;+}(\boldsymbol{x})\right)\right)=-\overline{\mathrm{N}}_{\infty}^{1 ; \pm} f(\boldsymbol{x}),
\end{aligned}
$$

so $f$ is odd, hence $\langle$ ie 1$\rangle$ is proved.

$\langle$ ie2 $\rangle \quad$ This statement follows directly from $\langle\mathrm{ks} 1\rangle$ of Theorem 6.4.

To prove $\langle$ ie 3$\rangle$ and $\langle$ ie 4$\rangle$, we chose an arbitrary point $\boldsymbol{y}_{1}=\Psi_{p}^{1 ;+}\left(\boldsymbol{x}_{0}\right) \in \hat{\mathcal{K}}_{1}^{n}$, so we have the sequence of points $\boldsymbol{y}_{i}$ given by recursion (8.3), hence the sequences $\boldsymbol{y}_{2 i+1}$ and $\boldsymbol{y}_{2 i}$ tend to points $O^{ \pm}$, respectively. (See Fig. 7.)

$\left\langle\right.$ ie3 We can assume $p<-1$ by the symmetry of $\mathcal{S}^{n}=\mathcal{K}_{1}^{n}$.
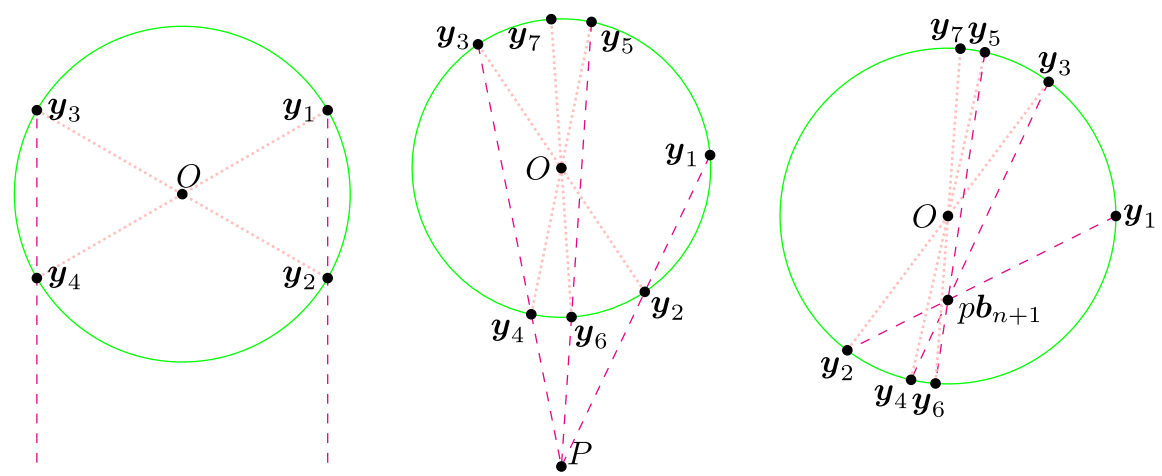

Fig. 7 The first points of (8.3) if $\kappa=1: p=\infty,|p|>1$, and $|p|<1$ 
Assume that $h \in C\left(\mathbb{K}_{1}^{n}, p\right)$ is in the kernel of $\mathrm{R}_{p}^{1}$. This means that the even function $\tilde{h} \in C\left(\mathcal{K}_{1}^{n}, p\right)$ is such that $\mathrm{F}_{p}^{1} \tilde{h}$ vanishes. As $|p|>1,\langle\mathrm{ks} 3\rangle$ of Theorem 6.4 gives a function $f \in C\left(\mathcal{M}_{1 ; p}^{n}\right)$ such that $\pm \tilde{h}_{p}^{ \pm} \circ \Psi_{p}^{1 ; \pm}=\overline{\mathbf{N}}_{p}^{1 ; \pm} f$.

Since $\tilde{h}$ is even, we have $\tilde{h}\left(\boldsymbol{y}_{2 i+1}\right) \stackrel{p}{=} \tilde{h}\left(\boldsymbol{y}_{2 i}\right)$ for every $i \in \mathbb{N}$. So, by (6.7) and (5.3), we get

$$
\frac{\tilde{h}\left(\boldsymbol{y}_{2 i+2}\right)}{\tilde{h}\left(\boldsymbol{y}_{2 i}\right)}=\frac{\tilde{h}\left(\boldsymbol{y}_{2 i+2}\right)}{\tilde{h}\left(\boldsymbol{y}_{2 i+1}\right)}=\frac{\tilde{h}_{p}^{-}\left(\boldsymbol{y}_{2 i+2}\right)}{\tilde{h}_{p}^{+}\left(\boldsymbol{y}_{2 i+1}\right)}=\frac{-\overline{\mathrm{N}}_{p}^{1 ;-} f\left(\boldsymbol{x}_{i}\right)}{\overline{\mathrm{N}}_{p}^{1 ;+} f\left(\boldsymbol{x}_{i}\right)}=-\frac{\left|v_{p}^{1 ;+}\left(\left|\boldsymbol{x}_{i}\right|\right)\right|^{n-1}}{\left|v_{p}^{1 ;-}\left(\left|\boldsymbol{x}_{i}\right|\right)\right|^{n-1}} .
$$

Let $\phi_{p}=\lim _{e \rightarrow 0}\left|\frac{\nu_{p}^{1 ;+}(e)}{v_{p}^{1 ;-}(e)}\right|$. Then (4.3) gives

$$
\phi_{p}=\lim _{e \rightarrow 0}\left|\frac{-p+\sqrt{1-e^{2}\left(p^{2}-1\right)}}{-p-\sqrt{1-e^{2}\left(p^{2}-1\right)}}\right|=\frac{-p+1}{|-p-1|}>1,
$$

and therefore

$$
\lim _{i \rightarrow \infty} \frac{\tilde{h}\left(\boldsymbol{y}_{2 i+4}\right)}{\tilde{h}\left(\boldsymbol{y}_{2 i}\right)}=\phi_{p}^{2(n-1)}>1 .
$$

Thus $\tilde{h}\left(\boldsymbol{y}_{2}\right) \neq 0$ implies that $\left|\tilde{h}\left(O^{-}\right)\right|=\infty$, a contradiction, hence $\tilde{h}\left(\boldsymbol{y}_{2}\right)$ vanishes which, as $\boldsymbol{y}_{2}$ was chosen arbitrarily, proves $\langle\mathrm{ie} 3\rangle$.

〈ie4〉 We only need to prove for $p \in(-1,0]$ by the symmetry of $\mathcal{S}^{n}=\mathcal{K}_{1}^{n}$.

Assume that $h \in C_{\infty}\left(\mathbb{K}_{1}^{n}, p\right)$ is in the kernel of $\mathrm{R}_{p}^{1}$. This means that the even function $\tilde{h} \in C_{\infty}\left(\mathcal{K}_{1}^{n}, p\right)$ is such that $\mathrm{F}_{p}^{1} \tilde{h}$ vanishes. As $|p|<1,\langle\mathrm{ks} 2\rangle$ of Theorem 6.4 gives a function $f \in C_{\infty}\left(\mathcal{M}_{1 ; p}^{n}\right)$ such that $\pm \tilde{h}_{p}^{ \pm} \circ \Psi_{p}^{1 ; \pm}=\overline{\mathrm{N}}_{p}^{1 ; \pm} f$.

If $p=0$, then $\pm \tilde{h}_{p}^{ \pm} \circ \Psi_{p}^{1 ; \pm}=\overrightarrow{\mathrm{N}}_{p}^{1 ; \pm} f$ shows that $\tilde{h}$ is odd, so, being also even, $\tilde{h}$ vanishes which proves $\langle$ ie 4$\rangle$.

Therefore we can assume $p \in(-1,0)$ from now on.

For any point $\boldsymbol{y}_{1}=\Psi_{p}^{1 ;+}\left(\boldsymbol{x}_{0}\right) \in \hat{\mathcal{K}}_{1}^{n}$, we can apply recursion (8.3) again to get the sequence of points. (See Fig. 7.) This again leads to (8.4), which implies $\tilde{h}\left(\boldsymbol{y}_{2}\right) \equiv 0$ in the same way as in the proof of $\langle$ ie 3$\rangle$. Since $\boldsymbol{y}_{2}$ was chosen arbitrarily, $\langle$ ie 4$\rangle$ follows.

The proof is complete.

Observe that Theorem 8.1 can be understood also as a result about pairs of shifted Funk transforms on the sphere [3-6], and $\langle\mathrm{ie} 4\rangle$ can be considered as a generalization of $\left[24,\left(\mathrm{i}^{+}\right)\right.$ of Theorem 3.2].

We put here the parabolic case only for the sake of a kind of completeness.

Theorem 8.2 The duplex Funk-type isodistant Radon transforms in the parabolic space have the following properties:

〈ip1〉 If $h \in C_{\infty}\left(\mathbb{K}_{0}^{n}\right)$, then $\mathrm{R}_{\infty}^{0} h$ vanishes if and only if $h$ is an odd function.

$\left\langle\right.$ ip2 $\quad$ If $p \in \mathbb{R}$, then $\mathbb{R}_{p}^{0}$ is injective on $C_{\infty}\left(\mathbb{K}_{0}^{n}\right)$.

We omit the proof because the reasoning behind $\langle\mathrm{ip} 1\rangle$ and $\langle\mathrm{ip} 2\rangle$ is very much similar to that of $\langle\mathrm{ie} 1\rangle$ and $\langle\mathrm{ie} 3\rangle$, respectively. In the other hand, in Sect. 9 we prove Theorem 9.3 that generalizes $\langle\mathrm{ip} 2\rangle$, which otherwise can be considered as a generalization of $\left[24,\left(\mathrm{i}^{+}\right)\right.$ of Theorem 3.2].

We turn to the hyperbolic case. 
Theorem 8.3 The duplex Funk-type isodistant Radon transforms in the hyperbolic space have the following properties:

〈ih1〉 $\quad \mathrm{R}_{0}^{-1}$ is injective on $C_{n}\left(\mathbb{K}_{-1}^{n}\right)$.

$\langle\mathrm{ih} 2\rangle \quad \mathrm{R}_{ \pm 1}^{-1}$ is injective on $C_{\infty}\left(\hat{\mathbb{K}}_{-1}^{n}, \pm 1\right)$.

〈ih3〉 If $h \in C_{\infty}\left(\mathbb{K}_{-1}^{n}\right)$, then $\mathrm{R}_{\infty}^{-1} h$ vanishes if and only if there is an odd function $f \in C\left(\mathcal{M}_{-1 ; \infty}^{n}\right)$ such that $\pm \tilde{h}_{\infty}^{ \pm} \circ \Psi_{\infty}^{-1 ; \pm}=\overline{\mathrm{N}}_{\infty}^{-1 ; \pm} f$, where $\tilde{h}=h \circ \chi_{\kappa^{*}}$.

〈ih4〉 Let $|p|>0,|p| \neq 1$, and let $h \in C\left(\mathbb{K}_{-1}^{n}, p\right)$ be such that $h\left(\operatorname{Exp}_{O^{+}}(\right.$eu $\left.)\right)$vanishes for every $e \geq s=|\ln | p||$ and $\boldsymbol{u} \in \mathcal{S}^{n-1}$. If $\mathrm{R}_{p}^{-1} h$ vanishes, then $h$ vanishes too.

Proof $\langle$ ih 1$\rangle$ Let $h \in C_{n}\left(\mathbb{K}_{-1}^{n}\right)$ be in the kernel of $\mathrm{R}_{0}^{-1}$. This means that the even function $\tilde{h} \in C_{n}\left(\mathcal{K}_{-1}^{n}\right)$ is such that $\mathrm{F}_{0}^{-1} \tilde{h}$ vanishes. Then $\langle\mathrm{kh} 2\rangle$ of Theorem 6.6 gives a function $f \in C\left(\mathcal{M}_{-1 ; 0}^{n}\right)$ such that $\pm \tilde{h}_{0}^{ \pm} \circ \Psi_{0}^{-1 ; \pm}=\overline{\mathbf{N}}_{0}^{-1 ; \pm} f$. This shows that $\tilde{h}$ is odd, so, being also even, $\tilde{h}$ vanishes that proves $\langle$ ih 1$\rangle$.

$\langle$ ih2 $\rangle$ This statement follows directly from $\langle\mathrm{kh} 1\rangle$ of Theorem 6.6. The proof of $\langle\mathrm{ih} 3\rangle$ is so much similar to the proof of $\langle\mathrm{ie} 1\rangle$ that we leave it to the readers' consideration.

〈ih4〉 Since $|p|>0$, the symmetry of $\mathcal{K}_{-1}^{n}$ allows us to assume $p<0$. With this assumption we also have $p \neq-1$, and $s=|\ln (-p)|$. Then the even function $\tilde{h} \in C_{n-1}\left(\mathcal{K}_{-1}^{n}\right)$ is such that $\mathrm{F}_{p}^{-1} \tilde{h}$ vanishes, hence $\langle\mathrm{kh} 3\rangle$ and $\langle\mathrm{kh} 4\rangle$ of Theorem 6.6 gives a function $f \in C\left(\mathcal{M}_{-1 ; p}^{n}\right)$ such that $\pm \tilde{h}_{p}^{ \pm} \circ \Psi_{p}^{-1 ; \pm}=\overline{\mathrm{N}}_{p}^{-1 ; \pm} f$.

Choose an arbitrary point $\boldsymbol{y}_{1}=\Psi_{p}^{-1 ;+}\left(\boldsymbol{x}_{0}\right) \in \hat{\mathcal{K}}_{-1}^{n}$ such that $d_{-1}\left(O^{+}, \boldsymbol{y}_{1}\right)<|\ln (-p)|$. Then the sequence of points $\boldsymbol{y}_{i}$ given by recursion (8.3), is such that sequences $\boldsymbol{y}_{2 i+1}$ and $\boldsymbol{y}_{2 i}$ tend to points $O^{+}$and $O^{-}$, respectively.

Since $\tilde{h}$ is even, we have $\tilde{h}\left(\boldsymbol{y}_{2 i+1}\right)=\tilde{h}\left(\boldsymbol{y}_{2 i}\right)$ for every $i \in \mathbb{N}$. So (5.3) and (4.3) lead to

$$
\frac{\tilde{h}\left(\boldsymbol{y}_{2 i+2}\right)}{\tilde{h}\left(\boldsymbol{y}_{2 i}\right)}=\frac{\tilde{h}\left(\boldsymbol{y}_{2 i+2}\right)}{\tilde{h}\left(\boldsymbol{y}_{2 i+1}\right)}=\frac{\tilde{h}_{p}^{-}\left(\boldsymbol{y}_{2 i+2}\right)}{\tilde{h}_{p}^{+}\left(\boldsymbol{y}_{2 i+1}\right)}=\frac{-\overline{\mathrm{N}}_{p}^{-1 ;-} f\left(\boldsymbol{x}_{i}\right)}{\overline{\mathrm{N}}_{p}^{-1 ;+} f\left(\boldsymbol{x}_{i}\right)}=-\frac{\left|\nu_{p}^{-1 ;+}\left(\left|\boldsymbol{x}_{i}\right|\right)\right|^{n-1}}{\left|\nu_{p}^{-1 ;-}\left(\left|\boldsymbol{x}_{i}\right|\right)\right|^{n-1}} .
$$

Let $\phi_{p}=\lim _{e \rightarrow 0}\left|\frac{v_{p}^{-1 ;+}(e)}{v_{p}^{-1 ;-}(e)}\right|$ Then (4.3) gives

$$
\phi_{p}=\lim _{e \rightarrow 0}\left|\frac{-p+\sqrt{1+e^{2}\left(p^{2}-1\right)}}{-p-\sqrt{1+e^{2}\left(p^{2}-1\right)}}\right|=\frac{-p+1}{|-p-1|}>1,
$$

and therefore

$$
\lim _{i \rightarrow \infty} \frac{\tilde{h}\left(\boldsymbol{y}_{2 i+4}\right)}{\tilde{h}\left(\boldsymbol{y}_{2 i}\right)}=\phi_{p}^{2(n-1)}>1 .
$$

Thus $\tilde{h}\left(\boldsymbol{y}_{2}\right) \neq 0$ implies that $\left|\tilde{h}\left(O^{-}\right)\right|=\infty$, a contradiction, hence $\tilde{h}\left(\boldsymbol{y}_{2}\right)$ vanishes that, as $\boldsymbol{y}_{2}$ was chosen arbitrarily, proves $\langle$ ih 4$\rangle$.

Notice that $\langle\mathrm{ih} 1\rangle$ is exactly $\left[24,\left(\mathrm{i}^{-}\right)\right.$of Theorem 3.2]. 


\section{Notices and discussion}

Pulling and applying other already known results perhaps most importantly the range descriptions through our intertwining relations (5.4) and (5.5) will lead to numerous new results about the shifted Funk transforms and, more importantly from our point of view, about the Funk-type isodistant Radon transforms.

The $k$-dimensional isodistants $k \leq n-1$ can be defined through the cross-sections of $\mathcal{K}_{\kappa}^{n}$ with the $k$-dimensional affine planes $(k \leq n-1)$. Then our intertwining relations extend to these $k$-dimensional isodistants, so the method of [24, Theorem 3.1] extends our support theorems to these $k$-dimensional isodistants, which would improve the decay conditions. This time we leave this for the future, but pay attention to [32], where this is done on the sphere for the 1-shifted Funk transform, i.e., for the spherical slice transform of Abouelaz-Daher-Helgason type.

The isodistant of a totally geodesic $\mathcal{G}^{k}$ of codimension $n-k \geq 1$ is like a tube of codimension 1 around $\mathcal{G}^{k}$. The associated Radon transform $\mathrm{R}_{\kappa}^{\mathbb{E}, k}$ gives the integral of every suitable function over every isodistant using the natural measure. This is quite a different kind of transformation, so our method seems to be unusable, hence its investigation remains to the future.

Combining the intertwining relations (5.4) for different values of $p$ but with the same curvature $\kappa$ leads to intertwining relations similar in spirit to those that are in $[3,4,6$, $7,20,26,30,33,34,38]$ for the sphere, but also for the hyperbolic case. For instance, we show here two relevant applications of this idea for the sphere $\mathcal{S}^{n}=\mathcal{K}_{1}^{n}$. Firstly, we improve $\langle\mathrm{ks} 2\rangle$ of Theorem 6.4 considerably.

Theorem 9.1 〈ks2'> If $|p|<1$ and $h \in C\left(\mathcal{S}^{n}\right)$, then $\mathrm{F}_{p}^{1} h$ vanishes if and only if $\mathrm{N}_{p}^{1 ;+}\left(h_{p}^{+} \circ \Psi_{p}^{1 ;+}\right)=-\mathrm{N}_{p}^{1 ;-}\left(h_{p}^{-} \circ \Psi_{p}^{1 ;-}\right)$.

Proof Since $|p|<1$, we have $\mathcal{M}_{1 ; p}^{n}=\mathbb{R}^{n}$ by (4.2). So, by (5.1), we have the mapping $\Psi_{p}^{1 ; \pm}: \mathbb{R}^{n} \rightarrow \mathcal{K}_{p}^{1 ; \pm}$ for every $p \in(-1,1)$, where $\mathcal{K}_{p}^{1 ; \pm}=\operatorname{Im} \Psi_{p}^{1 ; \pm}$ by $(5.2)$.

Let $h \in C\left(\mathcal{S}^{n}\right)$, and define $h_{p}^{ \pm}: \mathcal{K}_{p}^{\kappa ; \pm} \rightarrow \mathbb{R}$ by (6.7). Using $\overline{\mathrm{N}}_{p}^{1 ; \pm}$, given in (5.3), let $f_{p}^{ \pm}=\mathrm{N}_{p}^{1 ; \pm}\left(h_{p}^{ \pm} \circ \Psi_{p}^{1 ; \pm}\right)$.

Let $\tilde{h}_{r}^{ \pm}=\left(\overline{\mathrm{N}}_{r}^{1 ; \pm} f_{p}^{ \pm}\right) \circ \bar{\Psi}_{r}^{1 ; \pm}$, and define the function $\tilde{h}: \mathcal{S}^{n} \rightarrow \mathbb{R}$ by (6.8). Then $\tilde{h} \in C\left(\mathcal{S}^{n}\right)$ is clear, because $\lim _{t \rightarrow \infty} f_{p}^{ \pm}(t \boldsymbol{u}+P)=h\left(\boldsymbol{u}+p \boldsymbol{b}_{n+1}\right)$ for every point $P \in \mathbb{R}^{n}$ and $\boldsymbol{u} \in \mathcal{S}^{n-1}$.

Since Theorem 5.1 gives $\frac{\mathrm{S}_{ \pm}^{1} h_{p}^{ \pm}(p ; \boldsymbol{w}, q)}{\sqrt{1+q^{2}\left(1-p^{2}\right)}}=\operatorname{Rf}_{p}^{ \pm}(\boldsymbol{w}, q)=\frac{\mathrm{S}_{ \pm}^{1} h_{r}^{ \pm}(r ; \boldsymbol{w}, q)}{\sqrt{1+q^{2}\left(1-r^{2}\right)}}$, by (6.1) and (4.15), we obtain the intertwining relation

$$
\begin{aligned}
\mathrm{F}_{p}^{1} h(\boldsymbol{w}, q) \sqrt{1+q^{2}\left(1-r^{2}\right)} & =\left(\mathrm{S}_{+}^{1} h_{p}^{+}(p ; \boldsymbol{w}, q)+\mathrm{S}_{-}^{1} h_{p}^{-}(p ; \boldsymbol{w}, q)\right) \sqrt{1+q^{2}\left(1-r^{2}\right)} \\
& =\left(\mathrm{S}_{+}^{1} \tilde{h}_{r}^{+}(r ; \boldsymbol{w}, q)+\mathrm{S}_{-}^{1} \tilde{h}_{r}^{-}(r ; \boldsymbol{w}, q)\right) \sqrt{1+q^{2}\left(1-p^{2}\right)} \\
& =\mathrm{F}_{r}^{1} \tilde{h}(\boldsymbol{w}, q) \sqrt{1+q^{2}\left(1-p^{2}\right)} .
\end{aligned}
$$

Thus $\mathrm{F}_{p}^{1} h$ vanishes if and only if $\mathrm{F}_{r}^{1} \tilde{h}$ vanishes.

Letting $r=0$, we can use Funk's result [13] saying that $\mathrm{F}_{0}^{1} \tilde{h}$ vanishes if and only if $\tilde{h}$ is an odd function, i.e., $\tilde{h}_{r}^{+} \circ \Psi_{r}^{1 ;+}=-\tilde{h}_{r}^{-} \circ \Psi_{r}^{1 ;-}$. By the definition of $\tilde{h}_{r}^{ \pm}$this is equivalent to $\overline{\mathrm{N}}_{r}^{1 ;+} f_{p}^{+}=-\overline{\mathrm{N}}_{r}^{1 ;-} f_{p}^{-}$, i.e., $f_{p}^{+}=-f_{p}^{-}$.

By the definition of $f_{p}^{ \pm}$this completes the proof. 
Secondly, we prove as an example that only the zero is a common element of the kernels of two special shifted Funk transforms. This result can be easily extended to all pairs of the shifted Funk transforms, but, for the sphere, it is done in a more general manner in [3]. For the other spaces it is left to the future. Notice though, that if $p \cdot r=1$, then there are nonvanishing continuous functions $h$ for which $\mathrm{F}_{p}^{1} h=\mathrm{F}_{r}^{1} h \equiv 0$.

Theorem 9.2 If $|p|<1,|r|<1$, and $h \in C\left(\mathcal{S}^{n}\right)$, then $\mathrm{F}_{p}^{1} h=\mathrm{F}_{r}^{1} h \equiv 0$ if and only if $h \equiv 0$.

Proof Statement $\left\langle\mathrm{ks} 2{ }^{\prime}\right\rangle$ says that if $|p|<1$, then $\mathrm{F}_{p}^{1} h$ vanishes if and only if

$$
-h_{p}^{-}\left(\Psi_{p}^{1 ;-}(\boldsymbol{x})\right)=\overline{\mathbf{N}}_{p}^{1 ;-} \mathbf{N}_{p}^{1 ;+}\left(h_{p}^{+}\left(\Psi_{p}^{1 ;+}(\boldsymbol{x})\right)\right)=\left|\frac{p-\sqrt{1+\boldsymbol{x}^{2}\left(1-p^{2}\right)}}{p+\sqrt{1+\boldsymbol{x}^{2}\left(1-p^{2}\right)}}\right|^{n-1} h_{p}^{+}\left(\Psi_{p}^{1 ;+}(\boldsymbol{x})\right) .
$$

Assume $-1<p<r<1$. Starting from any point $\boldsymbol{y}_{1}=\Psi_{p}^{\kappa ;+}\left(\boldsymbol{x}_{0}\right) \in \hat{\mathcal{K}}_{p}^{1 ;+}$, the recursion

$$
\begin{array}{ll}
\boldsymbol{y}_{2 i+2}:=\Psi_{p}^{\kappa ;-}\left(\boldsymbol{x}_{2 i}\right), & \boldsymbol{x}_{2 i+1}:=\bar{\Psi}_{r}^{\kappa ;-}\left(\boldsymbol{y}_{2 i+2}\right), \\
\boldsymbol{y}_{2 i+3}:=\Psi_{r}^{\kappa ;+}\left(\boldsymbol{x}_{2 i+1}\right), & \boldsymbol{x}_{2 i+2}:=\bar{\Psi}_{p}^{\kappa ;+}\left(\boldsymbol{y}_{2 i+3}\right)
\end{array}
$$

generates points for every $i=1,2, \ldots$. This sequence of points $\boldsymbol{y}_{i}$, as it is easy to see on the rightmost illustration of Fig. 7, is infinite and the sequences $\boldsymbol{y}_{2 i+1}$ and $\boldsymbol{y}_{2 i}$ tend to the points $O^{ \pm}$, respectively. Since we have

$$
\lim _{i \rightarrow \infty} \frac{\tilde{h}\left(\boldsymbol{y}_{2 i+2}\right)}{\tilde{h}\left(\boldsymbol{y}_{2 i}\right)}=\left|\frac{r+1}{r-1} \frac{p-1}{p+1}\right|^{n-1}=\left|\frac{r+1}{p+1} \frac{1-p}{1-r}\right|^{n-1}>1,
$$

$h\left(\boldsymbol{y}_{2}\right) \neq 0$ implies that $\left|h\left(O^{-}\right)\right|=\infty$, a contradiction, hence $h\left(\boldsymbol{y}_{2}\right)$ vanishes which, as $\boldsymbol{y}_{2}$ was chosen arbitrarily, completes the proof.

The kernel descriptions for duplex Funk-type isodistant Radon transforms in Sect. 8 could be easily extended for functions in the $L^{1}$ space. For instance, in Theorem 8.1 one should consider a "small" compact spherical cap $\mathcal{Y}_{1}$ in $\mathcal{K}_{1}^{n}$ with center at $\boldsymbol{y}_{1}$ and show that the sequence of compact neighborhoods $\mathcal{Y}_{i}$ of $\boldsymbol{y}_{i}$ generated by the recursion (8.3) is such that $\lim _{i \rightarrow \infty} \frac{\mu\left(\mathcal{Y}_{2 i+4}\right)}{\mu\left(\mathcal{Y}_{2 i}\right)}=\rho_{p}^{-2}$, where $\mu$ denotes the canonical surface measure on the sphere $\mathcal{K}_{1}^{n}$.

In the Euclidean space every duplex Funk-type isodistant Radon transform of a suitable function $f$ is a sum of the Euclidean Radon transforms of $f$ at two different hyperplanes, i.e., $\mathrm{R}_{p}^{0} f(\boldsymbol{w}, t)=\mathrm{R} f(\boldsymbol{w},(1-p) t)+\mathrm{R} f(\boldsymbol{w},(1+p) t)$, where $p \in \mathbb{R}$ is a constant. Recalling the curves of the $(p, q)$-plane mentioned in (1.4), we obtain the freaky ${ }^{5}$ Radon-type transform

$$
f \mapsto \mathrm{R}^{\langle r\rangle} f(\boldsymbol{w}, t):=\mathrm{R} f\left(\boldsymbol{w}, t-r \sqrt{1+t^{2}}\right)+\mathrm{R} f\left(\boldsymbol{w}, t+r \sqrt{1+t^{2}}\right)
$$

if the curve is $r^{2}\left(1+q^{2}\right)=p^{2} q^{2}(r>0)$, and the horocyclic ${ }^{6}$ Radon-type transform

$$
f \mapsto \mathrm{R}^{\{\alpha\}} f(\boldsymbol{w}, t):=\mathrm{R} f(\boldsymbol{w}, \cot \alpha-t)+\mathrm{R} f(\boldsymbol{w}, \cot \alpha+t)
$$

if the curve is $q=\tan \alpha(\alpha \in(0, \pi / 2))$. The problem of the injectivity of these Radon-type transforms raises the question of

\footnotetext{
5 This term was used by Ungar for a very similar problem on the sphere in [37].

6 This term comes from the case of $\alpha=\pi / 4$ in the hyperbolic space.
} 
what kind of transforms $\mathrm{M}, \mathrm{N}$ of the Grassmann manifold of hyperplanes

make the Radon-type transform $\mathrm{R}^{\mathrm{M}, \mathrm{N}}: \mathcal{F} \ni f \mapsto \mathrm{R}^{\mathrm{M}, \mathrm{N}} f=(\mathrm{R} f) \circ \mathrm{M}+(\mathrm{R} f) \circ \mathrm{N}$

to an injectivity on a reasonably large function space $\mathcal{F}$ ?

For an instant partial answer, which also generalizes Theorem 8.2, we define the Radontype transform $\mathrm{R}^{v}: f \mapsto \mathrm{R}^{v} f(\boldsymbol{w}, t)=\mathrm{R} f\left(\boldsymbol{w}, v_{-} t\right)+\mathrm{R} f\left(\boldsymbol{w}, v_{+} t\right)$ for the nonzero vectors $v=\left(v_{-}, v_{+}\right) \in \mathbb{R}^{2}$.

Theorem 9.3 Let $f \in C_{\infty}\left(\mathbb{R}^{n}\right)$ and let $\boldsymbol{v}=\left(v_{-}, v_{+}\right) \neq(0,0)$. Assume that $\mathrm{R}^{v} f(\boldsymbol{w}, t)$ vanishes for every $t>1$ and $\boldsymbol{w} \in \mathcal{S}^{n-1}$.

(i) If either $0<\left|v_{-}\right|<\left|v_{+}\right|$or $v_{+}=0$ or $v_{-}=v_{+}$, then the support of $f$ is in the ball $\left|v_{-}\right| \mathcal{B}^{n}$.

(ii) If $v_{-}=-v_{+}$, then $f$ is an odd or even function outside the ball $\left|v_{-}\right| \mathcal{B}^{n}$ if $n$ is even or odd, respectively.

Proof Recall that $\mathrm{R} f(\boldsymbol{w}, c t)=c^{n-1} \mathrm{R} f_{c}(\boldsymbol{w}, t)$, where $f_{c}: \boldsymbol{x} \mapsto f(c \boldsymbol{x})$, hence $\mathrm{R}^{v} f=\mathrm{R}\left(v_{-}^{n-1} f_{v}\right.$ $+v_{+}^{n-1} f_{v_{+}}$), so Support Theorem 2.1 gives that $v_{-}^{n-1} f_{v_{-}}+v_{+}^{n-1} f_{v_{+}}$vanishes outside the unit ball.

If $0<\left|v_{-}\right|<\left|v_{+}\right|$, then we get that $f(\boldsymbol{y})=-\frac{v_{+}^{n-1}}{v_{-}^{n-1}} f\left(\frac{v_{+}}{v_{-}} \boldsymbol{y}\right)$ for $|\boldsymbol{y}|>\left|v_{-}\right|$. Since $\left|v_{+} / v_{-}\right|>1$, we deduce that $f(\boldsymbol{y})=\left(-\frac{v_{+}^{n-1}}{v_{-}^{n-1}}\right)^{k} f\left(\left(\frac{v_{+}}{v_{-}}\right)^{k} \boldsymbol{y}\right)$ for every $k \in \mathbb{N}$ and $|\boldsymbol{y}|>\mid v_{-}$. This proves $f(\boldsymbol{y})=0$, because $f$ satisfies the infinite decay condition that implies $\left(-\frac{v_{+}^{n-1}}{v_{-}^{n-1}}\right)^{k} f\left(\left(\frac{v_{+}}{v_{-}}\right)^{k} \boldsymbol{y}\right) \rightarrow 0$ as $k \rightarrow \infty$.

If $v_{+}=0$, then $v_{-} \neq 0$ and we get that $f\left(v_{-} \boldsymbol{x}\right)$ vanishes for $|\boldsymbol{x}|>1$.

If $v_{-}=v_{+}$, then $v_{-} \neq 0$ and we get that $f\left(v_{-} \boldsymbol{x}\right)$ vanishes for $|\boldsymbol{x}|>1$.

If $v_{-}=-v_{+}$, then $v_{\mp} \neq 0$ and we get that $f_{v_{-}}+(-1)^{n-1} f_{-v_{-}}$vanishes outside the unit ball. This implies that $f_{v_{-}}$is an odd or even function outside the unit ball if $n$ is even or odd, respectively.

Notice that 9.3 is a generalization of $\left[24,\left(\mathrm{i}^{+}\right)\right.$of Theorem 3.2].

The investigation of problem (9.4) remains to a later paper.

It is worth paying attention to the relations both the spherical and the hyperbolic slice transforms have to the weighted versions of the so-called boomerang transform [11, 23], which is in fact the dual of the Radon transform. These relations can be shown through the stereographic projections $\Pi_{\mp 1}$ of $\mathcal{K}_{\kappa}^{n}$.

Finally, we note that the inverses $\bar{\Psi}_{p}^{\kappa ; \pm}$ of the maps given in (5.1) create models of $\mathbb{K}_{\kappa}^{n}$ in $\mathbb{R}^{n}$. These models are mostly unknown, but the projective Cayley-Klein models [39] are created by $p=0$, essentially the conform Poincare models $[2,12]$ are created by $p= \pm 1$, and the Gans models [14] are created by $p= \pm \infty$. The corresponding projections $\bar{\Psi}_{p}^{\kappa ; \pm}$ are called gnomonic [41] if $p=0$, stereographic [42] if $p= \pm 1$, and orthogonal if $p= \pm \infty$, respectively.

Acknowledgements I am very much grateful to the organizers for inviting me to the " Conference on Modern Challenges in Imaging (In the Footsteps of Allan MacLeod Cormack On the Fortieth Anniversary of his Nobel Prize)" held in the week August 5-9 at the Tufts University, Medford, Massachusetts, where the idea of this paper was born due to the talk of Mark Agranovsky. The author thanks for the help of Viktor Vígh 
and József Kozma. I am indebted to Károly Böröczky, jr., for inviting me to the Rényi Institute for the academic year 2018-2019, and I thank the hospitality of the Rényi Institute.

Funding Open access funding provided by University of Szeged.

Open Access This article is licensed under a Creative Commons Attribution 4.0 International License, which permits use, sharing, adaptation, distribution and reproduction in any medium or format, as long as you give appropriate credit to the original author(s) and the source, provide a link to the Creative Commons licence, and indicate if changes were made. The images or other third party material in this article are included in the article's Creative Commons licence, unless indicated otherwise in a credit line to the material. If material is not included in the article's Creative Commons licence and your intended use is not permitted by statutory regulation or exceeds the permitted use, you will need to obtain permission directly from the copyright holder. To view a copy of this licence, visit http://creativecommons.org/licenses/by/4.0/.

\section{References}

1. Abouelaz, A., Daher, R.: Sur la transformation de Radon de la sphère $S^{d}$. Bull. Soc. Math. 121(3), 353-382 (1993). https://doi.org/10.24033/bsmf.2212. (French, with English and French summaries)

2. Alekseevskij, D.V., Vinberg, E. B., Solodovnikov, A.S.: Geometry of spaces of constant curvature, Geometry, II, Encyclopaedia Math. Sci., vol. 29, pp. 1-138. Springer, Berlin (1993) https://doi.org/10. 1007/978-3-662-02901-5_1

3. Agranovsky, M.: Non-central Funk-Radon transforms: single and multiple. J. Funct. Anal. 108701, 41-279 (2020). https://doi.org/10.1016/j.jfa.2020.108701

4. Agranovsky, M., Rubin, B: Non-geodesic spherical Funk transforms with one and two centers (2019), available at arxiv:1904.11457

5. Agranovsky, M., Rubin, B.: On single and paired shifted Funk transform, Cormack Conference (2019), available at https://math.tufts.edu/faculty/equinto/Cormack2019/AbstractBook.pdf

6. Agranovsky, M., Rubin, B.: On two families of Funk-type transforms (2019), available at arxiv: 1908.06794

7. Antipov, Y.A., Estrada, R., Rubin, B.: Method of analytic continuation for the inverse spherical mean transform in constant curvature spaces. J. Anal. Math. 118(2), 623-656 (2012). https://doi. org/10.1007/s11854-012-0046-y

8. Berenstein, C.A., Casadio Tarabusi, E., An inversion formula for the horocyclic Radon transform on the real hyperbolic space, Tomography, impedance imaging, and integral geometry (South Hadley, MA: Lectures in Applied mathematics, vol. 30, American Mathematical Society. Providence, RI 1994, pp. 1-6 (1993)

9. Bray, W.O., Rubin, B.: Radon transforms over lower-dimensional horospheres in real hyperbolic space. Trans. Am. Math. Soc. 372(2), 1091-1112 (2019). https://doi.org/10.1090/tran/7666

10. Cannon, J.W., Floyd, W.J., Kenyon, R.W.: Hyperbolic geometry, Flavors of geometry, R. Parry pp. 59-115 (1997)

11. Cormack, A.M., Quinto, E.T.: A Radon transform on spheres through the origin in $R^{n}$ and applications to the Darboux equation. Trans. Am. Math. Soc. 260(2), 575-581 (1980). https://doi.org/10. 2307/1998023

12. Coxeter, H.S.M.: Non-Euclidean Geometry. MAA Spectrum, 6th edn. Mathematical Association of America, Washington (1998)

13. Funk, P.: Über Flachen mit lauter geschlossenen geodatischen Linien. Math. Ann. 74(2), 278-300 (1913). https://doi.org/10.1007/BF01456044

14. Gans, D.: Classroom notes: a new model of the hyperbolic plane. Am. Math. Mon. 73(3), 291-295 (1966). https://doi.org/10.2307/2315350

15. Gelfand, I.M., Graev, M.I.: Integral transformations connected with line complexes in a complex affine space. Dokl. Akad. Nauk SSSR 138, 1266-1269 (1961). (Russian)

16. Gindikin, S., Reeds, J., Shepp, L.: Spherical tomography and spherical integral geometry, Tomography, impedance imaging, and integral geometry (South Hadley, MA: Lectures in Applied mathematics, vol. 30, American Mathematical Society, Providence, RI 1994, pp. 83-92 (1993)

17. Grinberg, E.L.: The admissibility theorem for the spatial X-ray transform over the twoelement field, The mathematical legacy of Leon Ehrenpreis, Springer Proceedings in Mathematics. Springer, Milan, vol. 16, pp. 111-123 (2012) https://doi.org/10.1007/978-88-470-1947-8_8 
18. Helgason, S.: Integral geometry and Radon transforms. Springer, New York (2011) https://doi.org/ 10.1007/978-1-4419-6055-9

19. Helgason, S.: Support theorems for horocycles on hyperbolic spaces. Pure Appl. Math. Q. 8(4), 921-927 (2012). https://doi.org/10.4310/PAMQ.2012.v8.n4.a4

20. Hielscher, R., Quellmalz, M.: Reconstructing a function on the sphere from its means along vertical slices. Inverse Prob. Imaging 10(3), 711-739 (2016). https://doi.org/10.3934/ipi.2016018

21. Hsiang, W.-I.: On the laws of trigonometries of two-point homogeneous spaces. Ann. Glob. Anal. Geom. 7(1), 29-45 (1989). https://doi.org/10.1007/BF00137400

22. Katsevich, A.: An inversion formula for the dual horocyclic Radon transform on the hyperbolic plane. Math. Nachr. 278(4), 437-450 (2005). https://doi.org/10.1002/mana.200310251

23. Kurusa, A.: New unified Radon inversion formulas. Acta Math. Hungar. 60(3-4), 283-290 (1992). https://doi.org/10.1007/BF00051646

24. Kurusa, A.: Support theorems for totally geodesic Radon transforms on constant curvature spaces. Proc. Am. Math. Soc. 122(2), 429-435 (1994). https://doi.org/10.2307/2161033

25. Palamodov, V.: Reconstruction from Integral Data, Chapman and Hall/CRC Monographs and Research Notes in Mathematics. Chapman and Hall/CRC, New York (2016). https://doi.org/10. 1201/b19575

26. Quellmalz, M.: A generalization of the Funk. Radon transform. Inverse Prob. 33(3), 035016 (2017). https://doi.org/10.1088/1361-6420/33/3/035016

27. Quellmalz, M.: The Funk-Radon transform for hyperplane sections through a common point (2018), available at arxiv: $1810.08105 \mathrm{v} 1$

28. Rubin, B.: Introduction to Radon Transforms (with Elements of Fractional Calculus and Harmonic Analysis), Encyclopedia of Mathematics and its Applications, vol. 160. Cambridge University Press, New York (2015)

29. Rubin, B.: New inversion formulas for the horospherical transform. J. Geom. Anal. 27(1), 908-946 (2017). https://doi.org/10.1007/s12220-016-9704-0

30. Rubin, B.: Reconstruction of functions on the sphere from their integrals over hyperplane sections. Anal. Math. Phys. (2019). https://doi.org/10.1007/s13324-019-00290-1

31. Rubin, B.: The vertical slice transform on the unit sphere. Fract. Calc. Appl. Anal. 22(4), 899-917 (2019). https://doi.org/10.1515/fca-2019-0049

32. Rubin, B.: On the spherical slice transform, pp. 899-917 (2021), available at arxiv:2101.06783

33. Salman, Y.: An inversion formula for the spherical transform in $S^{2}$ for a special family of circles of integration. Anal. Math. Phys. 6(1), 43-58 (2016). https://doi.org/10.1007/s13324-015-0105-5

34. Salman, Y.: Recovering functions defined on the unit sphere by integration on a special family of subspheres. Anal. Math. Phys. 7(2), 165-185 (2017). https://doi.org/10.1007/s13324-016-0135-7

35. Salman, Y.: Recovering Functions Defined on $S^{n-1}$ by Integration on Subspheres Obtained from Hyperplanes Tangent to a Spheroid, (2017), available at arxiv:1704.00349v1

36. Schneider, R.: Functions on a sphere with vanishing integrals over certain subspheres. J. Math. Anal. Appl. 26, 381-384 (1969). https://doi.org/10.1016/0022-247X(69)90160-7

37. Ungar, P.: Freak theorem about functions on a sphere. J. Lond. Math. Soc. 29, 100-103 (1954). https:// doi.org/10.1112/j1ms/s1-29.1.100

38. Zangerl, G., Scherzer, O.: Exact reconstruction in photoacoustic tomography with circular integrating detectors II: spherical geometry. Math. Methods Appl. Sci. 33(15), 1771-1782 (2010). https://doi.org/ 10.1002/mma.1266

39. Wikipedia, Beltrami-Klein model, https://en.wikipedia.org/wiki/Beltrami\%E2\%80\%93Klein_model, Last accessed on October 17 (2019)

40. Wikipedia, Poincaré disk model, https://en.wikipedia.org/wiki/Poincar\%C3\%A9_disk_model, Last accessed on October 17 (2019)

41. Wikipedia, Gnomonic projection, https://en.wikipedia.org/wiki/Gnomonic_projection, Last accessed on October 17 (2019)

42. Wikipedia, Stereographic projection, https://en.wikipedia.org/wiki/Stereographic_projection, Last accessed on October 17 (2019)

Publisher's Note Springer Nature remains neutral with regard to jurisdictional claims in published maps and institutional affiliations. 